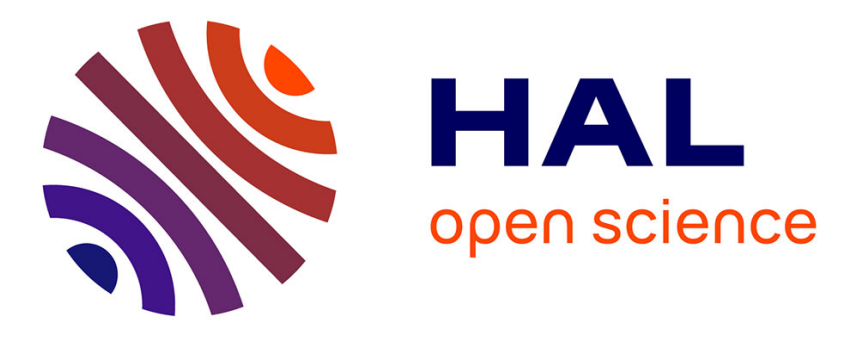

\title{
L'évolution des inégalités sociales de tabagisme au cours du cycle de vie: une analyse selon le sexe et la génération
}

Damien Bricard, Florence Jusot, François Beck, Myriam Khlat, Stéphane Legleye

\section{To cite this version:}

Damien Bricard, Florence Jusot, François Beck, Myriam Khlat, Stéphane Legleye. L'évolution des inégalités sociales de tabagisme au cours du cycle de vie: une analyse selon le sexe et la génération. Economie et Statistique / Economics and Statistics, 2015, 475-476. hal-01523696

\section{HAL Id: hal-01523696 https://hal.science/hal-01523696}

Submitted on 16 May 2017

HAL is a multi-disciplinary open access archive for the deposit and dissemination of scientific research documents, whether they are published or not. The documents may come from teaching and research institutions in France or abroad, or from public or private research centers.
L'archive ouverte pluridisciplinaire HAL, est destinée au dépôt et à la diffusion de documents scientifiques de niveau recherche, publiés ou non, émanant des établissements d'enseignement et de recherche français ou étrangers, des laboratoires publics ou privés. 


\title{
L'évolution des inégalités sociales de tabagisme au cours du cycle de vie : une analyse selon le sexe et la génération
}

\author{
Damien Bricard, Florence Jusot, François Beck, \\ Myriam Khlat et Stéphane Legleye *
}

Comment évoluent les inégalités sociales dans la consommation de tabac en France selon l'âge et les générations ? Afin de répondre à cette question, les données du Baromètre santé recueillies en 2010 auprès de 27653 personnes ont été mobilisées pour reconstruire de façon rétrospective le parcours tabagique de trois cohortes de naissance (1941-1955, 1956-1970 et 1971-1985). L'évolution des inégalités de tabagisme est étudiée à partir de la comparaison des prévalences du tabagisme calculées à chaque âge selon le niveau de diplôme, le sexe et la génération puis à l'aide d'un indice relatif d'inégalité estimé à l'aide de régressions logistiques.

Les résultats montrent qu'après avoir été plus fréquent parmi les plus diplômés, le tabagisme a reculé dans ces milieux, alors qu'il continue de progresser parmi les groupes moins diplômés. Cette analyse confirme également le décalage d'une génération chez les femmes par rapport aux hommes dans la diffusion du tabagisme, même si les niveaux de prévalence sont aujourd'hui forts pour les deux sexes. L'importance des inégalités sociales face au tabagisme ressort aux âges jeunes, pour toutes les cohortes et pour les deux sexes. Pour les cohortes les plus anciennes, les inégalités s'amenuisent au cours de la vie jusqu'à s'inverser chez les femmes. Pour la cohorte la plus récente, les inégalités restent à un niveau élevé tout au long du cycle de vie et tendent à s'accentuer après 25 ans pour les femmes.

Rappel :

Les jugements et opinions exprimés par les auteurs n'engagent qu'eux mêmes, et non les institutions auxquelles ils appartiennent, ni a fortiori l'Insee.
Sur la base de ce constat, il pourrait être efficace de cibler les politiques de prévention du tabagisme selon les groupes sociaux et selon les âges de la vie, et en particulier d'axer les efforts sur la prévention de l'entrée dans le tabagisme dans les milieux les moins diplômés.

Codes JEL : I12, D63, D91, C23.

Mots clés : inégalités, tabagisme, évolution, génération, cycle de vie.

* appartenances respectives : Ined; PSL, Université Paris-Dauphine, LEDa-LEGOS et Ined; OFDT, Inpes et Cermes 3 ; Ined ; Ined et Inserm U669.

Ce travail a été réalisé dans le cadre du projet COTCEDIS "Consommation de tabac et de cannabis : évolution et dynamiques de construction des inégalités sociales » ( $n^{\circ}$ 2011-250) financé par l'Institut national du cancer. Nous remercions l'Inpes pour la mise à disposition des données du Baromètre santé 2010. 
$\mathbf{L}$ es comportements à risque et en particulier le tabagisme comptent parmi les causes les plus importantes de morbidité et de mortalité évitable (Khaw et al., 2008). Le tabagisme est également plus fréquent dans les milieux les moins favorisés, en France (Peretti Watel et al., 2008) comme dans les autres pays européens (Mackenbach et al., 2008) et il contribue fortement aux inégalités sociales de santé (par exemple Balia et Jones, 2008 ; Balia et Jones, 2011 ; Mackenbach et al., 2011; Stringhini et al., 2010; Cutler et al., 2011 ; Tubeuf et al., 2012). Mesurer les inégalités sociales face au tabagisme et comprendre les dynamiques en jeu sont des étapes essentielles de la définition des politiques de réduction du tabagisme et plus généralement de réduction des inégalités de santé.

La littérature économique a proposé différents mécanismes pour expliquer la relation entre la situation sociale et le tabagisme (Braakman, 2011 ; Cutler et Lleras-Muney, 2010 ; Pampel, 2009). L'éducation améliorerait tout d'abord l'état de santé à la fois par une meilleure culture sanitaire mais aussi par une meilleure capacité à utiliser ces connaissances pour investir en santé. Les plus favorisés seraient par ailleurs incités à investir dans leur santé dans la mesure où le coût d'opportunité lié aux événements de santé augmente avec le salaire, ce qui expliquerait une initiation moins fréquente ou un arrêt plus précoce pour ceux qui ont commencé à fumer. De même, une espérance de vie plus élevée dans ces milieux les incite à s'investir plus pour maintenir un bon état de santé. Cet effet protecteur du salaire peut cependant être compensé par un effet de contrainte budgétaire qui rend plus accessible la consommation de tabac aux plus favorisés. En dernier lieu, les inégalités sociales de tabagisme pourraient être liées à des facteurs expliquant à la fois le tabagisme et la situation sociale tels que le milieu social d'origine, les préférences pour le risque ou le présent, les normes sociales ou des facteurs génétiques et cognitifs. Ces différents facteurs sont susceptibles de contribuer aux évolutions des inégalités sociales de tabagisme avec l'âge mais aussi à travers les générations.

Plusieurs travaux se sont intéressés aux inégalités sociales de tabagisme à différents étapes du cycle de vie et en particulier aux différences selon le niveau d'éducation. Ces travaux ont établi que chez les adolescents, le passage de l'expérimentation à l'usage quotidien était plus fréquent parmi les jeunes issus de milieux sociaux modestes ou défavorisés (Etilé, 2007 ; Legleye et al., 2011a ; Beck et Amsellem-Mainguy, 2012). Il est apparu également, à partir de données rétrospectives recueillies en population adulte, que ce passage plus fréquent était associé à un arrêt de consommation moins fréquent parmi les personnes les moins éduquées (Balia et Jones, 2008 ; Balia et Jones, 2011; Bricard et Jusot, 2012 ; Etilé et Jones, 2011 ; Federico et al., 2007 ; Fernandez et al., 2001 ; Jefferis et al., 2004 ; Legleye et al., 2011b ; Maralani, 2013 ; Schiaffino et al., 2003). Les conclusions de ces travaux suggèrent ainsi que les inégalités sociales se construisent peu à peu lors du passage de l'adolescence à l'âge adulte, les disparités existant à l'entrée étant renforcées par les disparités de sortie du tabagisme. Cependant, aucune étude n'a véritablement exploré la dynamique des inégalités sociales de tabagisme selon 1'âge.

En dehors de la dynamique des inégalités de tabagisme au cours du cycle de vie, l'évolution des inégalités de tabagisme avec les générations est également utile pour comprendre le cadre général de constitution des inégalités dans la société. Celle-ci peut être analysée dans le cadre théorique de l'« épidémie tabagique », une notion qui conceptualise le processus d'adoption de la consommation de tabac en fonction de l'âge, du niveau d'éducation, du sexe des individus et des générations (Lopez et al., 1994). Ce processus repose sur la théorie de la diffusion des innovations sociales (Rogers et Shoemaker, 1971), selon laquelle les groupes sociaux favorisés ouvrent la voie en acquérant des biens ou des comportements qui les différencient et les distinguent mais qui se diffusent ensuite vers les groupes les moins favorisés sur le principe de l'imitation. Par la suite, ce sont également les groupes favorisés qui réduisent en premier leur consommation, au fur et à mesure que l'information sur les dangers du tabac se répand dans la population. La prévalence du tabagisme dans les groupes sociaux serait donc fonction du moment d'observation, de leur place dans la hiérarchie des positions sociales, déterminant leur position de « pionnier » ou de « suiveur », et de leurs connaissances et de leurs sensibilités aux messages sanitaires. Ce cadre théorique prédit aussi une diffusion du tabagisme plus tardive chez les femmes que chez les hommes au fur et à mesure de leur émancipation (Waldron, 1991) même si les modalités de diffusion et en particulier le décalage de calendrier entre les sexes peuvent varier selon les pays (Thun et al., 2012). 
En France, les inégalités de tabagisme semblent plus fortes chez les hommes que chez les femmes, et des travaux récents ont établi un accroissement des inégalités parmi les générations les plus récentes, aussi bien en matière de passage à l'usage quotidien qu'en matière d'arrêt de la consommation (Legleye et al., 2011b; Peretti Watel et al., 2009). Ce schéma suggère que la France se situe, comme la plupart des pays européens, dans la dernière phase du cycle de diffusion : la consommation s'abaisse d'abord dans les groupes sociaux favorisés et ensuite dans les moins favorisés. Le processus de diffusion sociale des pratiques chez les femmes présenterait en outre un décalage de plusieurs décennies par rapport aux hommes (Legleye et al., 2011b ; Nourrisson, 1999). Toutefois, ces études considèrent successivement l'entrée puis la sortie éventuelle du tabagisme, et ne s'attachent pas à décrire le niveau de consommation de tabac des groupes sociaux qui résulte de la prise en compte simultanée des initiations et des arrêts. Ces travaux ne se sont penchés que sur les différences moyennes de tabagisme entre groupes sociaux et selon les cohortes sans véritablement étudier la dynamique d'évolution des inégalités sociales de tabagisme au cours du cycle de vie dans différentes générations.

L'objectif de cette étude est de développer une analyse descriptive de la dynamique par âge des inégalités (sociales et selon le sexe) de consommation de tabac au sein de trois générations (ou cohortes) successives à partir des histoires tabagiques recueillies dans le Baromètre santé 2010 (cf. encadré). La démarche proposée fournit une perspective sur l'ensemble du cycle de vie des individus de chaque cohorte et permet de comparer les évolutions de la proportion de fumeurs à chaque âge entre les niveaux de diplôme, cela pour différentes cohortes. Elle permet ainsi de distinguer des effets d'âge et de génération à partir d'une enquête transversale. Toutes les analyses seront menées séparément pour les hommes et les femmes, afin de rendre compte des disparités et des dynamiques différenciées selon le sexe. En décrivant l'évolution des inégalités de consommation de tabac dans une perspective historique couvrant les six dernières décennies, ce travail vise à fournir des éléments de compréhension précieux pour la définition des politiques de prévention et de lutte contre le tabagisme.

Dans un premier temps, nous présentons une description de l'évolution de la prévalence du tabagisme aux différents âges selon le sexe et la génération. Nous comparons ensuite ces dynamiques de tabagismes selon le niveau de diplôme. Nous proposons enfin une analyse paramétrique des inégalités de tabagisme selon le diplôme, le sexe et la génération fondée sur un indicateur relatif d'inégalité.

\section{Une approche en deux temps permettant de déceler l'évolution des inégalités de tabagisme avec l'âge pour différentes générations}

L'objectif de cet article est de mesurer l'évolution des inégalités sociales dans la prévalence du tabagisme selon l'âge pour différentes générations. Il existe un débat sur la mesure de l'évolution des inégalités sociales et en particulier sur les caractéristiques des indicateurs à utiliser (Mackenbach et Kunst, 1997 ; Wagstaff et al., 1991). Nous avons choisi de mesurer le milieu socioéconomique sur la base du niveau d'éducation afin d'avoir un indicateur stable au cours du cycle de vie qui possède la caractéristique d'être à la fois un reflet des conditions de vie dans l'enfance mais aussi d'être un déterminant majeur de la situation sociale au cours du cycle de vie. Nous nous intéresserons d'abord à l'évolution des prévalences selon le niveau d'éducation comme indicateur de la position sociale afin de décrire l'évolution des inégalités de tabagisme liées au niveau de diplôme. Nous mesurerons ensuite les évolutions par le biais d'une variable de rang liée au niveau de diplôme, pour prendre en compte l'évolution de la distribution des diplômes avec les générations en classant les individus au sein de cette distribution par génération.

Nous proposons deux étapes pour cette analyse. Dans un premier temps, la reconstruction des parcours tabagiques rétrospectifs à partir de l'âge à l'entrée dans l'usage quotidien du tabac et de l'âge à l'arrêt de la consommation nous permet de décrire les parcours tabagiques à chaque âge rétrospectif $a$ pour chacune des cohortes puis pour les différents niveaux d'éducation. Compte tenu des âges différents au moment de l'enquête des enquêtés des différentes cohortes, il est possible de décrire le parcours tabagique rétrospectif entre 14 et 39 ans pour la cohorte 1971-1985, entre 14 et 54 ans pour la cohorte 1956-1970 et entre 14 et 69 ans pour la cohorte 1941-1955. Ceci se fait par le calcul de la proportion de fumeurs quotidiens à chaque âge rétrospectif $a$ parmi les personnes présentes à cet âge au sein de la base personne-année. Ces proportions ont été représentées sur des graphiques. 
Il est aussi possible de calculer le nombre d'années de tabagisme quotidien pour chaque individu de chaque cohorte. La moyenne du nombre d'années de tabagisme pour une cohorte donnée (ou un niveau de diplôme donné) est estimée par la somme des proportions de fumeur à chaque âge rétrospectif pour cette même cohorte. Cette analyse est complétée par une comparaison des

Encadré

\section{LES DONNÉES}

Cette étude repose sur les données du Baromètre santé 2010, enquête réalisée par l'Institut national de prévention et d'éducation pour la santé (Inpes) [ $n=27653]$. II s'agit d'une enquête téléphonique quinquennale conduite régulièrement depuis 1992 mais dont la méthodologie a évolué afin de s'adapter progressivement à la complexité de l'offre en équipement (Beck et al., 2011). Elle repose sur un sondage aléatoire à deux degrés (ménage puis individu) et est représentative de la population francophone âgée de 15 à 75 ans résidant en métropole. Le questionnaire est multithématique mais centré sur les comportements de santé.

En 2010, le questionnaire permet de dater (rétrospectivement) l'âge à l'entrée dans l'usage quotidien de tabac ("À quel âge avez-vous commencé à fumer régulièrement/tous les jours ? ") et l'âge lors de l'arrêt ("Depuis combien de temps avez-vous arrêté de fumer? »). Ces âges sont ainsi dits « rétrospectifs ».

Le parcours tabagique des enquêtés peut ainsi être représenté sous la forme d'une base personne-année permettant des analyses en temps discret. L'enquêté a autant d'observations que de périodes allant de sa quatorzième année à la date de l'enquête, date à laquelle il atteint l'âge $A$. On notera a l'âge rétrospectif qui correspond à l'âge de l'enquêté au sein de son parcours de vie rétrospectif et annais son année de naissance. Le parcours tabagique des enquêtés est ensuite traduit sous la forme d'une variable dichotomique représentant le statut de fumeur (oui/non) à chaque âge rétrospectif $\left(S_{i a}\right)$ à l'aide des âges d'entrée et d'arrêt de la consommation de tabac.

Trois cohortes définies à partir de la date de naissance des enquêtés sont considérées : la cohorte 1941-1955, la cohorte 1956-1970 et la cohorte 1971-1985.

Le milieu socioéconomique des individus est apprécié par le plus haut niveau de diplôme atteint. Le niveau de diplôme a été catégorisé en 4 groupes distinguant les enquêtés sans diplôme ou ayant été jusqu'au certificat d'études primaires d'une part, les enquêtés ayant réalisé des études de premier cycle ou de second cycle d'autre part (baccalauréat compris), les enquêtés ayant effectué une ou deux années d'études supérieures au baccalauréat ensuite, et enfin les enquêtés ayant réalisé au moins trois années d'études supérieures au baccalauréat. Ces catégories sont basées sur la classification internationale des diplômes de l'Organisation de coopération et de développement économique (OCDE, 1999).

Les statistiques descriptives de l'échantillon par niveau de diplôme sont présentées dans le tableau ci-dessous. Elles montrent la diminution très nette entre les générations des individus ayant au mieux un niveau d'études primaires avec une proportion qui passe de $36 \%$ pour les hommes et de $46 \%$ pour les femmes de la génération 1941-1955 à moins de $20 \%$ pour les hommes et pour les femmes de la génération 1971-1985, tandis que la proportion d'individus ayant un niveau de diplôme supérieur au baccalauréat est très nettement croissante, en particulier pour les femmes.

\section{Tableau}

Description de l'échantillon par niveau de diplôme selon la cohorte de naissance et le sexe

\begin{tabular}{|l|c|c|c|c|c|c|}
\hline \multirow{2}{*}{ Niveau de diplôme } & \multicolumn{2}{|c|}{ Cohorte 1941-1955 } & \multicolumn{2}{c|}{ Cohorte 1956-1970 } & \multicolumn{2}{c|}{ Cohorte 1971-1985 } \\
\cline { 2 - 7 } & Homme & Femme & Homme & Femme & Homme & Femme \\
\cline { 2 - 7 } & $(\mathrm{n}=2790)$ & $(\mathrm{n}=3848)$ & $(\mathrm{n}=3286)$ & $(\mathrm{n}=3925)$ & $(\mathrm{n}=3249)$ & $(\mathrm{n}=3842)$ \\
\hline Non scolarisés ou CEP & 35,7 & 46,1 & 26,4 & 30,0 & 17,8 & 18,7 \\
Études de premier ou de second cycle & 43,2 & 37,4 & 51,0 & 47,9 & 50,3 & 42,1 \\
1 ou 2 années d'études supérieures & 8,3 & 6,8 & 10,4 & 11,1 & 14,9 & 18,2 \\
au baccalauréat & & & & & & \\
3 ou + années d'études supérieures au baccalauréat & 12,8 & 9,7 & 12,2 & 11,0 & 17,0 & 21,0 \\
\hline
\end{tabular}

L'échantillon est restreint aux enquêtés d'au moins 25 ans au moment de l'enquête, dont le niveau d'études peut être considéré comme définitif, et de moins de 70 ans de façon à minimiser les biais de sélection dus à la mortalité. Trois groupes de générations seront analysés séparément: la génération des enquêtés nés entre 1941 et 1955 (âgés de 55 à 69 ans au moment de l'enquête), la génération des enquêtés nés entre 1956 et 1970 (âgés de 40 à 54 ans) et la génération des enquêtés nés de 1971 à 1985 (âgés de 25 à 39 ans). 
prévalences hommes-femmes du tabagisme au cours de la vie afin d'apprécier les dynamiques propres aux deux sexes.

Dans une deuxième étape, afin d'étudier plus finement la dynamique de construction de ces inégalités en fonction de l'âge rétrospectif, nous proposons d'étudier les inégalités de tabagisme liées au niveau de diplôme à l'aide d'un indicateur synthétique : l'indice relatif d'inégalité ou relative index of inequality (Mackenbach et Kunst, 1997 ; Hayes, 2002). Cet indicateur repose sur le classement des individus selon des groupes de statut socio-économique (ici le diplôme), du plus avantagé ou moins avantagé. Ce classement permet alors de construire une variable de rang qui, pour une variable catégorielle ordonnée telle que le niveau de diplôme, correspond à la proportion d'individus ayant au moins ce niveau augmentée de la moitié de la proportion de personnes ayant exactement ce niveau. Cette variable, comprise entre 0 et 1 , est une fonction décroissante du niveau de diplôme ${ }^{1}$. Elle peut être traitée comme une variable continue, l'écart entre les valeurs reflétant la distribution des diplômes correspondants.

L'intérêt de cette variable de rang pour notre étude est multiple. D'abord, elle prend en considération l'ensemble de la distribution des diplômes dans la population à un moment donné, et ne se contente pas d'offrir la comparaison entre deux niveaux de diplômes donnés : le caractère commun et inégalitaire de la répartition des diplômes est pris en compte. Ensuite, elle permet de s'affranchir dans une large part de la dévalorisation des diplômes en tenant compte de l'évolution de leur distribution dans le temps. Pour ce faire, la variable de rang a été calculée séparément pour chaque sexe et pour des groupes correspondant à chaque année de naissance. Enfin, elle permet de simplifier et de synthétiser la présentation des résultats de l'analyse en ne représentant que la courbe de la valeur de l'indicateur d'inégalité à chaque âge, permettant de rendre compte des inégalités liées à la position sociale au cours du cycle de vie.

L'indice d'inégalité s'obtient ensuite par régression logistique d'une indicatrice de l'événement considéré sur la variable de rang. L'odds-ratio associé à la variable de rang s'interprète alors comme le sur-risque de tabagisme associé au plus faible rang d'éducation par rapport au rang le plus élevé et constitue la mesure de l'inégalité, que l'on nommera RII par la suite. Cet indicateur quantifie les inégalités éducationnelles de tabagisme de manière relative, c'est-à-dire sous la forme d'un rapport des chances (ou odds-ratio) entre la personne la moins diplômée et la personne la plus diplômée. Nous privilégions ici un indicateur relatif d'inégalité pour décrire l'évolution des inégalités avec l'âge plutôt qu'un indicateur absolu dans la mesure où celui-ci permet une analyse qui n'est pas dépendante de l'évolution de la prévalence moyenne avec l'âge. L'évolution au cours du cycle de vie des inégalités de tabagisme pour un sexe et une cohorte donnés peut être modélisée à l'aide d'un modèle estimant à chaque âge rétrospectif $a$ la probabilité d'être fumeur. Nous supposons ainsi que, au sein de chaque cohorte, une variable latente $S_{i a}^{*}$ représente la propension à fumer de l'individu $i$ à l'âge rétrospectif $a$ et peut s'écrire comme suit :

$$
\begin{aligned}
& S_{i a}^{*}=\alpha \cdot \text { annais }_{i}+\beta_{a} \cdot \text { rang }_{i} \cdot I_{a}+\gamma_{a} \cdot I_{a}+\varepsilon_{i a} \\
& \forall a=14, \ldots, A
\end{aligned}
$$

Avec $S_{i a}=1$ si $S_{i a}^{*}>0$ et $S_{i a}=0$ si $S_{i a}^{*} \leq 0$

Le coefficient $\alpha$ associé à la variable annais $_{i}$ (introduite en variable continue) capture le différentiel de tabagisme en fonction de l'année de naissance au sein de la cohorte étudiée, le vecteur de coefficients $\beta_{a}$ mesure l'effet de la variable de rang pour chaque indicatrice du vecteur d'âge rétrospectif $I_{a}$, et le coefficient $\gamma_{a}$ mesure le différentiel de tabagisme associé au vecteur d'âge rétrospectif $I_{a}$. Ce modèle permet ainsi de tester la présence d'une interaction entre la variable de rang et l'âge rétrospectif $a$ par le test de l'égalité des coefficients $\beta_{a}$ et de déterminer ainsi s'il y a une évolution des inégalités de tabagisme avec l'âge.

Six modèles sont donc estimés, un par cohorte et par sexe. Dans chacun de ces modèles, chaque individu est observé autant de fois qu'il est présent dans la cohorte rétrospective (de 14 ans à 39 ans ou plus selon son âge au moment de l'enquête). Ainsi, les observations successives d'un même individu ne sont pas indépendantes. Pour tenir compte de l'interdépendance des observations pour chaque individu, nous estimons un modèle logistique avec une correction de l'hétéroscédasticité par cluster individuel. Pour des raisons de clarté des représentations graphiques,

\footnotetext{
1. Par exemple, pour les hommes de la cohorte 1941-1955, le rang a pour valeur 0,06 pour 3 années ou plus d'études supérieures au baccalauréat, 0,17 pour 1 ou 2 années d'études supérieures au baccalauréat, 0,43 pour le premier ou second cycle et 0,82 pour les personnes non scolarisées ou n'ayant que le CEP. Ces rangs sont calculés à partir du tableau de l'encadré.
} 
les intervalles de confiance à $95 \%$ des estimations des odds-ratios ne figurent pas toujours sur les graphiques.

Toutes les estimations proposées (prévalences, durées moyennes de tabagisme quotidien, odds-ratios etc.) sont effectuées en partie sur des données censurées. Par exemple, seule une partie des individus de la cohorte 1971-1985 ont atteint 39 ans au moment de l'enquête : les autres ne sont observés que jusqu'à un âge inférieur. Le même phénomène vaut pour l'observation de la cohorte 1956-1970 pour les âges rétrospectifs compris entre 40 et 54 ans et pour la cohorte 1941-1955 pour les âges rétrospectifs compris entre 55 et 69 ans. À titre d'information, pour la cohorte $1971-1985,50 \%$ des individus ne sont plus observés à partir de l'âge rétrospectif de 33 ans; pour les cohortes 1956-1970 et 1941-1955, ces âges médians valent respectivement 47 et 61 ans. Toutes les estimations tiennent compte de cette censure.

\section{Chez les hommes comme chez les femmes, le profil des prévalences selon l'âge s'apparente à une courbe en cloche}

Chez les hommes la prévalence du tabagisme a légèrement diminué avec les générations, alors que celle-ci a augmenté fortement chez les femmes entre la génération 1941-1955 et la génération 1956-1970 avant de fléchir légèrement pour la génération 1971-1985 (cf. graphique I).

\section{Graphique I}

Prévalence du tabagisme en fonction de l'âge - hommes et femmes des cohortes 1941-1955, 1956-1970 et 1971-1985
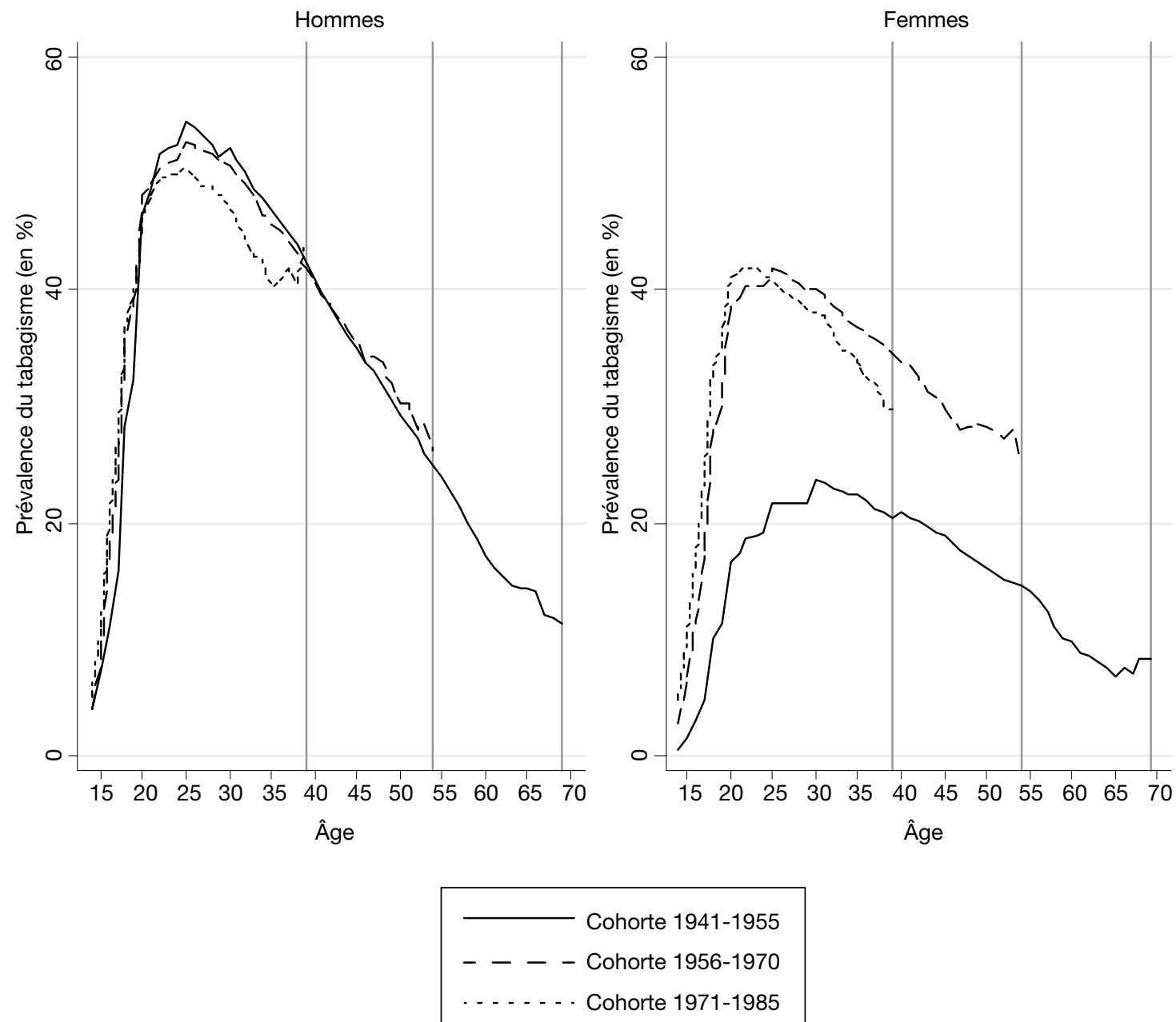

Lecture : les prévalences du tabagisme en fonction de l'âge rétrospectif sont calculées selon la cohorte et le sexe par reconstruction des parcours tabagiques.

Champ : population française, hommes et femmes âgés de 25 à 69 ans au moment de l'enquête (âgés de 14 à 39 ans pour la cohorte 1971-1985, de 40 à 54 ans pour la cohorte 1956-1970 et de 55 à 69 ans pour la cohorte 1941-1955).

Source : Inpes, Baromètre santé 2010. 
Quel que soit la génération, le profil global des prévalences selon l'âge est semblable à une courbe en cloche. La prévalence du tabagisme augmente rapidement de 14 ans à 20 ans et continue de progresser lentement jusqu'à 25-30 ans. Elle diminue ensuite continûment. Ce profil est assez proche chez les hommes et les femmes (bien que les niveaux diffèrent). Parmi les enquêtés de la cohorte 1941-1955, $54 \%$ des hommes et $22 \%$ des femmes fumaient à 25 ans. Dans la cohorte 1956-1970, le tabagisme concernait $53 \%$ des hommes et $42 \%$ des femmes à 25 ans, et dans la cohorte 1971-1985, $51 \%$ des hommes et $41 \%$ des femmes à 25 ans. Le profil tabagique selon l'âge a tout de même connu une légère évolution entre toutes les cohortes chez les hommes et entre les deux dernières cohortes chez les femmes : les prévalences tabagiques ont cru plus rapidement avant l'âge de 20 ans alors qu'elles ont décru plus rapidement pour les cohortes les plus récentes après l'âge de 25 ans. Parmi les hommes, l'âge au pic de consommation n'a pas sensiblement varié entre les cohortes (environ 25 ans), et on note un net rajeunissement parmi les femmes (de 30 ans pour la cohorte 1941-1955 à 25 ans pour la cohorte 1956-1970 et 22 ans pour la cohorte 1971-1985).

\section{Les prévalences tabagiques des hommes et des femmes tendent à se rapprocher}

Une analyse plus approfondie des différences entre hommes et femmes confirme un rapprochement des prévalences tabagiques masculines et féminines entre la plus ancienne et la plus récente cohorte avec une évolution de l'odds-ratio global associé au sexe masculin de 3,0 pour la cohorte $1941-1955$, à 1,4 pour la cohorte 1956-1970 et 1,3 pour la cohorte 1971-1985. L'évolution de la sur-prévalence masculine en fonction de l'âge a aussi été différente selon la cohorte, ainsi qu'il résulte de l'examen de l'odds-ratio associé au sexe masculin en fonction de l'âge rétrospectif pour les trois cohortes (cf. graphique II). Dans la cohorte 1941-1955, la sur-prévalence masculine décroît d'abord rapidement avec l'âge, jusqu'à 20 ans environ, puis repart à la hausse jusqu'à atteindre un pic local à 23 ans avant de redécroître continûment aux âges ultérieurs. Cela s'explique non seulement par une initiation plus tardive des femmes mais également par une décrue relativement plus prononcées chez les hommes que chez les femmes des prévalences tabagiques après l'âge de 25 ans. Le pic local intermédiaire à 23 ans pourrait, quant à lui, refléter un abandon probable de la cigarette par les femmes au moment des grossesses. Dans la cohorte 1956-1970, la sur-prévalence masculine connaît une très légère diminution avec l'âge qui reflète là aussi un rythme de sortie du tabagisme plus fort chez les hommes que chez les femmes. On ne discerne pas de pic local notable de sur-masculinité. Dans la cohorte 1971-1985, l'évolution de la sur-prévalence masculine avec l'âge est inversée et croît légèrement avec l'âge. Les prévalences masculines et féminines ne sont pas différentes jusqu'à l'âge de 16 ans puis évoluent progressivement avec l'âge en faveur des hommes jusqu'à 25 ans, âge auquel l'écart entre les sexes présente un plateau jusqu'à 35 ans, avant de repartir à la hausse. Ce dernier résultat suggère qu'après une initiation similaire, les femmes cessent à présent de fumer plus précocement que les hommes.

L'évolution des prévalences tabagiques au fil des générations et le rapprochement des prévalences des hommes et des femmes peut aussi être résumée par l'évolution des durées de tabagisme qui en résulte. La durée moyenne du tabagisme entre 14 et 39 ans a ainsi diminué de 10,9 années pour la cohorte 1941-1955 à 10,6 années pour la cohorte 1971-1985 chez les hommes alors qu'elle est passée de 4,5 années à 8,7 années sur la même période d'âge pour ces mêmes cohortes chez les femmes (cf. tableau 1).

\section{Dans la génération la plus ancienne, les femmes les plus éduquées étaient les plus concernées par le tabagisme...}

Le graphique III présente la prévalence du tabagisme quotidien aux différents âges rétrospectifs, selon le niveau de diplôme, pour chaque sexe et pour les trois générations considérées, nées respectivement en 1941-1955, en 1956-1970 et en 1971-1985.

Pour la génération la plus ancienne, les résultats montrent un gradient social peu marqué chez les hommes, et un gradient social inverse chez les femmes. Ainsi, chez les hommes, les personnes ayant au mieux un diplôme de l'enseignement primaire sont plus fréquemment fumeurs que les autres alors que les différences sont très faiblement marquées entre les autres niveaux d'études. À 25 ans, $57 \%$ des hommes non scolarisés ou diplômés de l'enseignement primaire étaient fumeurs alors qu'ils étaient $53 \%$ chez les plus diplômés. Ce gradient est observé chez les hommes jusqu'à l'âge de 40 ans et diminue ensuite jusqu'à s'annuler à partir de 
45 ans. Aux âges plus élevés, on observe une légère inversion du gradient qui témoigne d'un arrêt du tabagisme plus important à partir de la soixantaine des hommes avec un plus faible niveau d'études. Cette inversion du gradient chez les hommes de plus de 60 ans peut être

\section{Graphique II \\ Odds-ratio associé au sexe masculin pour le tabagisme quotidien en fonction de l'âge pour les cohortes 1941-1955, 1956-1970 et 1971-1985}
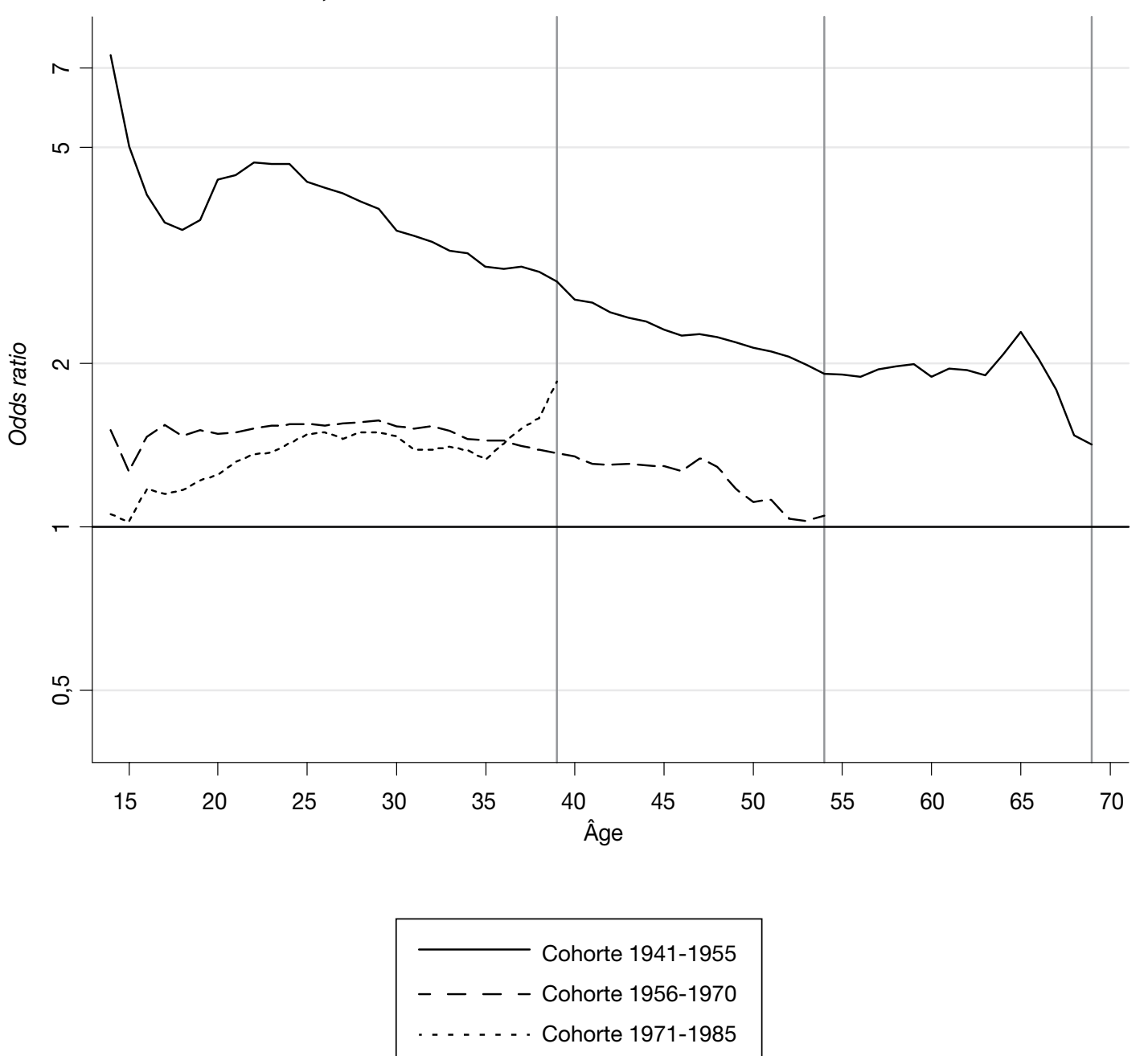

Lecture : les odds-ratios associés au sexe masculin pour la consommation quotidienne de tabac (représentés à l'échelle logarithmique sur ce graphique) sont issus de modélisations logistiques séparées par cohorte; ils sont estimés par interaction entre l'âge rétrospectif et le sexe de l'enquêté. Une valeur supérieure à 1 indique une surconsommation masculine.

Champ : population française, hommes et femmes âgés de 25 à 69 ans au moment de l'enquête (âgés de 14 à 39 ans pour la cohorte 1971-1985, de 40 à 54 ans pour la cohorte 1956-1970 et de 55 à 69 ans pour la cohorte 1941-1955).

Source : Inpes, Baromètre santé 2010.

Tableau 1

\section{Durée moyenne du tabagisme selon la tranche d'âge, la cohorte et le sexe}

En années

\begin{tabular}{|l|c|c|c|c|c|c|}
\hline \multirow{2}{*}{ Tranche d'âge } & \multicolumn{2}{|c|}{ Cohorte 1941-1955 } & \multicolumn{2}{c|}{ Cohorte 1956-1970 } & \multicolumn{2}{c|}{ Cohorte 1971-1985 } \\
\cline { 2 - 6 } & Homme & Femme & Homme & Femme & Homme & Femme \\
\hline Entre 14 et 39 ans & 10,9 & 4,5 & 11,0 & 8,7 & 10,6 & \\
Entre 14 et 54 ans & 15,8 & 7,2 & 16,1 & 13,1 & \\
Entre 14 et 69 ans & 18,3 & 8,6 & & & \\
\hline
\end{tabular}

Lecture : la durée moyenne du tabagisme est calculée par addition des prévalences rétrospectives moyennes par cohorte et par sexe sur les différentes tranches d'âge considérées.

Champ : population française, hommes et femmes âgés de 25 à 69 ans au moment de l'enquête (âgés de 14 à 39 ans pour la cohorte 1971-1985, de 40 à 54 ans pour la cohorte 1956-1970 et de 55 à 69 ans pour la cohorte 1941-1955).

Source : Inpes, Baromètre santé 2010. 
liée à des arrêts plus fréquents chez les moins éduqués pour cause de mauvaise santé à cet âge. Chez les femmes, les résultats ne montrent pas de différences sociales très nettes jusqu'à l'âge de 18 ans. Il apparaît ensuite un gradient social inversé très marqué, les femmes de niveau d'études universitaires étant plus fréquemment fumeuses que les femmes ayant au mieux un diplôme du secondaire. À 25 ans, $18 \%$ des femmes non scolarisées ou avec un diplôme de l'enseignement primaire fumaient alors qu'elles étaient $30 \%$ à fumer chez les plus diplômées. Ce gradient social inversé prévaut jusqu'aux âges plus élevés.

\section{...mais ensuite, la désaffection des plus diplômées par rapport au tabac s'est étendue des hommes aux femmes}

Pour la génération 1956-1970, un gradient social plus marqué apparaît chez les hommes et l'on observe une inversion du gradient chez les femmes par rapport aux résultats observés pour la génération précédente. À chaque âge, la proportion de fumeurs quotidiens diminue avec le niveau d'études, bien que l'on n'observe pas de différences selon le niveau de diplôme parmi les personnes diplômées du supérieur. L'accentuation de ce gradient chez les hommes
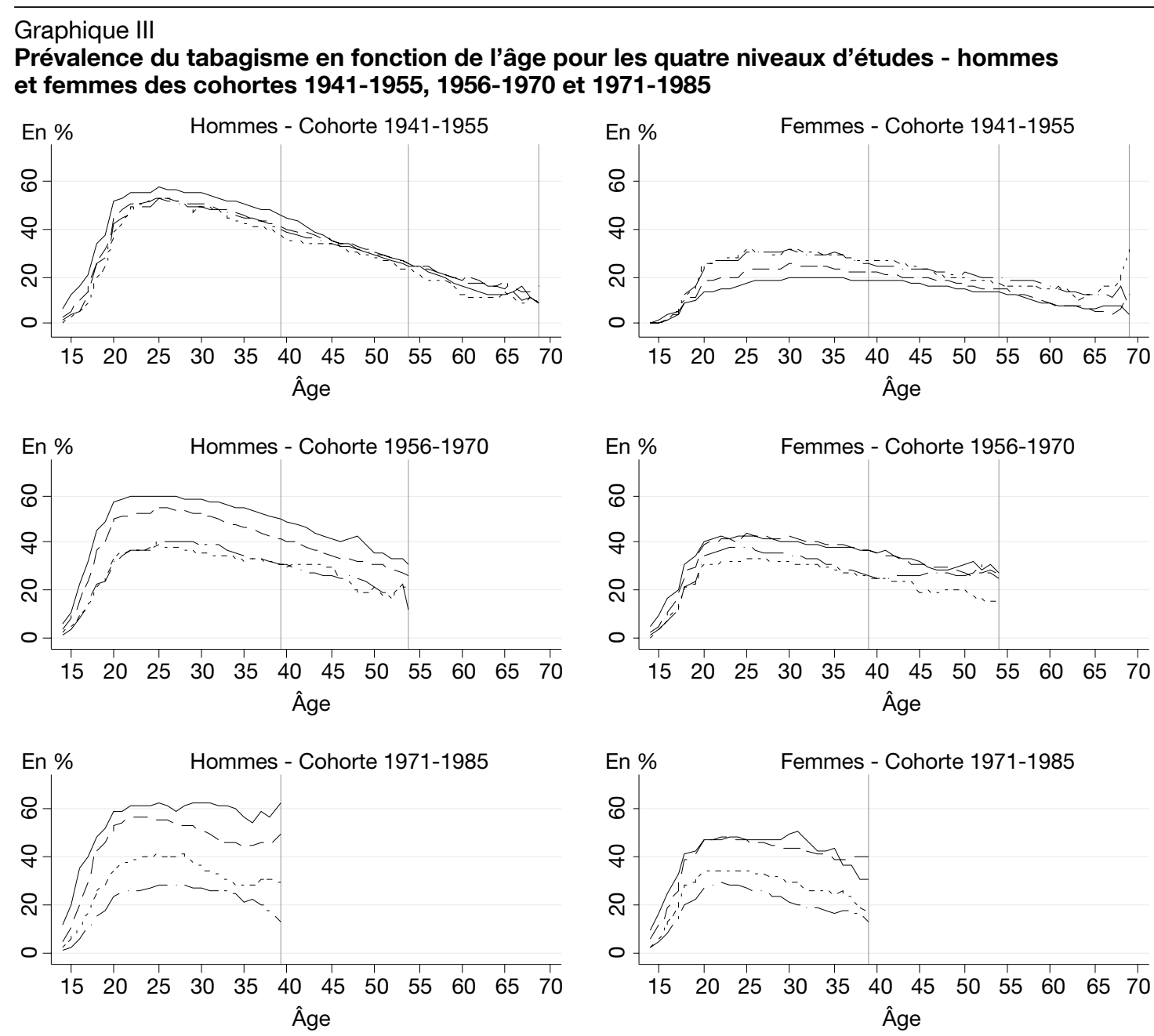

$$
\begin{aligned}
& \text { Non scolarisés ou CEP } \\
& \text { - }- \text { Études de premier ou de second cycle } \\
& \ldots \ldots+1 \text { ou } 2 \text { années d'études supérieures au baccalauréat } \\
& \ldots--3 \text { années ou plus d'études supérieures au baccalauréat }
\end{aligned}
$$

Lecture : les prévalences du tabagisme en fonction de l'âge rétrospectif sont calculées par cohorte et par sexe par reconstruction des parcours tabagiques selon le niveau de diplôme.

Champ : population française, hommes et femmes âgés de 25 à 69 ans au moment de l'enquête (âgés de 14 à 39 ans pour la cohorte 1971-1985, de 40 à 54 ans pour la cohorte 1956-1970 et de 55 à 69 ans pour la cohorte 1941-1955).

Source : Inpes, Baromètre santé 2010. 
est principalement due à un net recul des prévalences des hommes diplômés du supérieur par rapport à la génération précédente. Ainsi, à $25 \mathrm{ans}$, la prévalence des fumeurs parmi les plus diplômés était de 40 \% alors qu'elle est restée de $60 \%$ pour les moins diplômés. Chez les femmes, ce gradient semble en cours d'inversion dans la mesure où l'on observe seulement deux groupes distincts (les individus ayant au plus le baccalauréat et ceux ayant un diplôme de l'enseignement supérieur). À la différence des hommes, l'évolution du gradient chez les femmes provient principalement d'une nette hausse de la prévalence tabagique des moins diplômées par rapport à la génération précédente alors que la prévalence des plus diplômées n'a que très légèrement augmenté. Ainsi, à 25 ans, $43 \%$ des moins diplômées fumaient alors qu'elles étaient $38 \%$ à fumer chez les plus diplômées.

Au sein de la génération 1971-1985, on observe un gradient social très net chez les hommes et les femmes, la proportion de fumeurs étant continûment décroissante avec le niveau de diplôme, et ce, même chez les plus jeunes. Le gradient s'est principalement accentué chez les hommes par une diminution de la prévalence tabagique des plus éduqués qui atteint $28 \%$ à 25 ans et par une augmentation de la prévalence des moins éduqués qui atteint $63 \%$ à 25 ans. Le même mouvement est à l'œuvre chez les femmes : la prévalence des plus diplômées s'abaisse à $28 \%$ à 25 ans alors qu'elle s'élève à $48 \%$ pour les moins diplômées.

L'évolution des prévalences à chaque âge est résumée par la durée moyenne du tabagisme selon le niveau d'études (cf. tableau 2). Entre la cohorte 1941-1955 et la cohorte 1971-1985, la durée moyenne du tabagisme entre 14 et 39 ans pour les personnes non scolarisées ou diplômées au plus du certificat d'études primaires est passée de 11,8 années à 14,2 années pour les hommes et de 3,9 années à 10,6 années pour les femmes. Dans le même temps, on observe une convergence des comportements tabagiques masculins et féminins chez les personnes ayant poursuivi leurs études au moins 3 années au-delà du baccalauréat avec une diminution de la durée du tabagisme de 10,2 années à 5,5 années pour les hommes et une légère inflexion de 6,0 années à 5,2 années pour les femmes.

Tableau 2

Durée moyenne du tabagisme selon le niveau d'études, la tranche d'âge, la cohorte et le sexe

\begin{tabular}{|c|c|c|c|c|c|c|c|}
\hline \multirow[b]{2}{*}{ Niveau de diplôme } & \multirow[t]{2}{*}{ Cohorte } & \multicolumn{2}{|c|}{$1941-1955$} & \multicolumn{2}{|c|}{$1956-1970$} & \multicolumn{2}{|c|}{ 1971-1985 } \\
\hline & & Homme & Femme & Homme & Femme & Homme & Femme \\
\hline \multicolumn{8}{|c|}{ Non scolarisés ou certificat d'études primaires } \\
\hline Entre 14 et 39 ans & & 11,8 & 3,9 & 13,0 & 9,3 & \multirow[t]{3}{*}{14,2} & \multirow[t]{3}{*}{10,6} \\
\hline Entre 14 et 54 ans & & 16,9 & 6,2 & 19,1 & 14,0 & & \\
\hline Entre 14 et 69 ans & & 19,2 & 7,5 & & & & \\
\hline \multicolumn{8}{|c|}{ Premier ou second cycle } \\
\hline Entre 14 et 39 ans & & 10,5 & 4,7 & 11,3 & 9,1 & \multirow[t]{3}{*}{11,8} & \multirow[t]{3}{*}{10,3} \\
\hline Entre 14 et 54 ans & & 15,5 & 7,4 & 16,3 & 13,7 & & \\
\hline Entre 14 et 69 ans & & 18,2 & 8,8 & & & & \\
\hline \multicolumn{8}{|c|}{1 ou 2 années d'études supérieures } \\
\hline Entre 14 et 39 ans & & 9,9 & 6,1 & 7,9 & 6,8 & \multirow[t]{3}{*}{8,0} & \multirow[t]{3}{*}{6.8} \\
\hline Entre 14 et 54 ans & & 14,4 & 9,3 & 11,6 & 9,9 & & \\
\hline Entre 14 et 69 ans & & 16,4 & 11,7 & & & & \\
\hline \multicolumn{8}{|c|}{3 années d'études supérieures ou plus } \\
\hline Entre 14 et 39 ans & & 10,2 & 6,0 & 8,1 & 7,5 & \multirow[t]{3}{*}{5,5} & \multirow[t]{3}{*}{5,2} \\
\hline Entre 14 et 54 ans & & 15,0 & 9,3 & 11,7 & 11,5 & & \\
\hline Entre 14 et 69 ans & & 17,6 & 11,5 & & & & \\
\hline
\end{tabular}

Lecture : la durée moyenne du tabagisme est calculée par addition des prévalences rétrospectives moyennes par cohorte et par sexe sur les différentes tranches d'âge considérées selon le niveau d'études.

Champ : population française, hommes et femmes âgés de 25 à 69 ans au moment de l'enquête (âgés de 14 à 39 ans pour la cohorte 1971-1985, de 40 à 54 ans pour la cohorte 1956-1970 et de 55 à 69 ans pour la cohorte 1941-1955).

Source : Inpes, Baromètre santé 2010. 


\section{Les différences hommes-femmes demeurent au fil des générations plus prononcées chez les moins diplômés}

Le graphique IV représente le ratio des prévalences hommes-femmes du tabagisme au cours de la vie en fonction du niveau de diplôme pour les trois générations.

Pour la génération 1941-1955, les différences de prévalences hommes-femmes sont particulièrement prononcées en faveur des hommes et ces différences sont d'autant plus importantes que le niveau de diplôme est faible. Cependant, les écarts entre sexes sont quasiment identiques au sein des deux catégories de diplômés de l'enseignement supérieur. Les différences atteignent un rapport de chances de 5 en faveur des hommes pour les diplômés aux âges jeunes. Ainsi, dans la génération la plus ancienne, les hommes encouraient 5 fois plus de chances d'être fumeur que les femmes. Les différences de prévalences entre les hommes et les femmes décroissent jusqu'à l'âge de 39 ans. À 39 ans, un homme ayant au mieux un niveau d'étude primaire a environ 4 fois plus de chances d'être fumeur qu'une femme du même niveau d'études tandis qu'un homme diplômé du supérieur à environ 2 fois plus de chance d'être fumeur à cet âge qu'une femme diplômée du supérieur. Ces différences

\section{Graphique IV}

Odds-ratio associé au sexe masculin pour le tabagisme quotidien en fonction de l'âge pour les quatre niveaux d'études des cohortes 1941-1955, 1956-1970 et 1971-1985
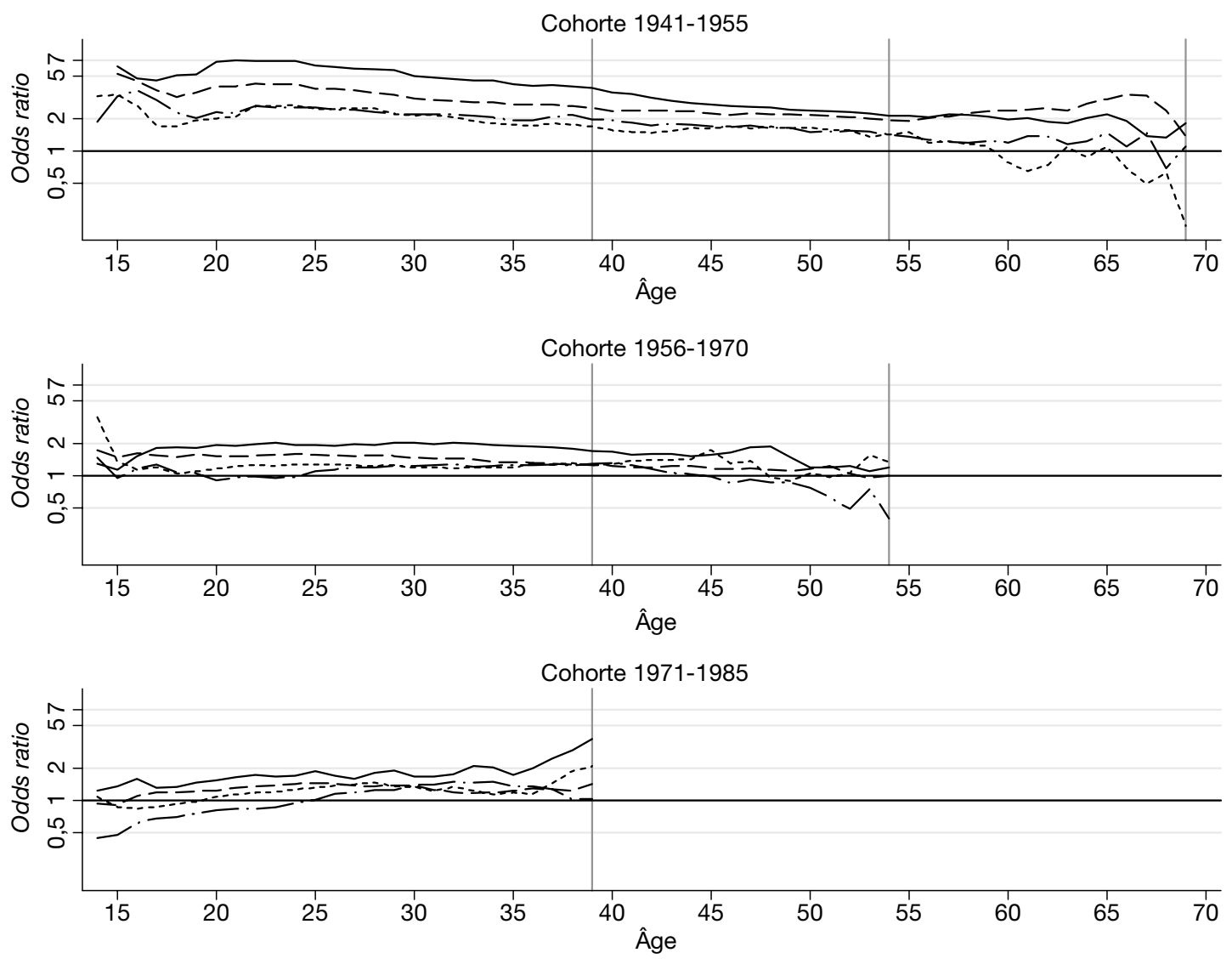

Non scolarisés ou CEP

- - - Études de premier ou de second cycle

-. . . . 1 ou 2 années d'études supérieures au baccalauréat

-.-. 3 années ou plus d'études supérieures au baccalauréat

Lecture : les odds-ratios associés au sexe masculin pour la consommation quotidienne de tabac représentés sur ce graphique (échelle logarithmique) sont issus de modélisations logistiques par cohorte et par niveau d'études ; ils sont estimés par interaction entre l'âge rétrospectif et le sexe de l'enquêté. Une valeur supérieure à 1 indique une surconsommation masculine.

Champ : population française, hommes et femmes âgés de 25 à 69 ans au moment de l'enquête (âgés de 14 à 39 ans pour la cohorte 1971-1985, de 40 à 54 ans pour la cohorte 1956-1970 et de 55 à 69 ans pour la cohorte 1941-1955).

Source : Inpes, Baromètre santé 2010. 
entre hommes et femmes diminuent ensuite avec l'âge, en étant toujours moins importantes chez les plus éduqués. Après 1'âge de 55 ans, les prévalences des hommes et des femmes diplômés du supérieur se rapprochent et vont même jusqu'à devenir plus importantes pour les femmes parmi les personnes avec une ou deux années d'études supérieures au baccalauréat.

Pour la génération 1956-1970, l'écart de prévalence au profit des hommes est d'autant plus élevé que le niveau de diplôme est faible, mais avec une amplitude bien moindre que pour la génération plus ancienne. Ainsi, entre 15 et 20 ans, un homme ayant au mieux un niveau d'études primaires a environ 1,5 fois plus de chances d'être fumeur qu'une femme du même niveau d'études. Les différences hommes-femmes pour les personnes ayant des niveaux d'études supérieurs au baccalauréat sont très faibles, et on observe même entre 15 et 25 ans à peu près la même prévalence pour les hommes et les femmes ayant poursuivi au moins 3 années d'études après le baccalauréat. À partir de 25 ans, les différences de prévalence diminuent jusqu'à atteindre un ratio de 1,2 , quel que soit le niveau d'études, jusqu'à l'âge de 39 ans. Après l'âge de 45 ans, on observe même une inversion du ratio entre les hommes et les femmes chez les diplômés du supérieur, parmi lesquels les femmes ont une prévalence du tabagisme quotidien supérieur à celui des hommes.

Pour la dernière génération née entre 1971 et 1985, les résultats sont nettement plus contrastés. Ils témoignent globalement du rapprochement très net des prévalences du tabagisme entre hommes et femmes, mais avec des trajectoires différentes selon le niveau de diplôme. Parmi les diplômés du supérieur, les femmes sont plus fréquemment fumeuses que les hommes avant 20 ans, et ce schéma se maintient jusqu'à 25 ans pour les femmes ayant fait 3 années ou plus d'études supérieures. Après cet âge, les hommes sont plus fréquemment fumeurs que les femmes, et ce quel que soit le diplôme. L'allure globale des courbes est donc celle d'une légère mais continue orientation à la hausse, qui tranche sur celles observées pour les générations antérieures. Cette évolution des inégalités entre hommes et femmes avec l'âge peut sans doute s'expliquer par un arrêt du tabac plus fréquent chez les femmes à l'approche de la première maternité. En effet, l'âge moyen auquel survient cette naissance est supérieur à 25 ans depuis les années 1980 et n'a cessé d'augmenter depuis pour atteindre
30 ans en 2009 (Pison, 2010). Pour les niveaux d'études inférieurs ou équivalents au baccalauréat, la sur-prévalence du tabagisme des hommes est stable jusqu'à l'âge de 35 ans avec un ratio des prévalences de 1,2 et augmente ensuite pour atteindre un ratio de prévalences supérieur à 2,0 à 39 ans pour le niveau d'études le plus bas.

\section{Les inégalités de tabagisme selon le diplôme se sont accrues d'une générations à l'autre}

Afin d'étudier plus finement la dynamique des inégalités de tabagisme liées au niveau d'éducation en fonction de l'âge, de la cohorte, et du genre, des indices relatifs d'inégalités (RII) ont été calculés à chaque âge rétrospectif, pour les 3 cohortes et selon le sexe. Ces indices correspondent aux odds ratios de l'effet associés à la variable de rang liée au niveau de diplôme à chaque âge rétrospectif (cf. annexe, tableaux A et B).

Ces résultats présentés dans le graphique $\mathrm{V}$ confirment en premier lieu que les inégalités de tabagisme selon le niveau d'éducation se sont globalement accrues entre les cohortes pour les hommes et pour les femmes, chez qui elles se sont mêmes inversées. L'indice relatif d'inégalités (RII) global entre les âges 14 et 39 ans est de 1,4 pour les hommes et de 0,6 pour les femmes de la cohorte 1941-1955, de 3,0 pour les hommes et de 1,7 pour les femmes de la cohorte 1956-1970 et de 6,9 pour les hommes et de 4,0 pour les femmes de la cohorte 1971-1985. Entre 14 et 39 ans, ces inégalités présentent une évolution significative avec l'âge pour toutes les cohortes sauf la cohorte intermédiaire chez les hommes (cf. annexe, tableau C : tests de l'égalité des coefficients).

Chez les hommes, ce RII est très élevé jusqu'à 18 ans, ce qui souligne l'importance des inégalités aux âges jeunes, et ce, pour les trois cohortes considérées. Au-delà de cet âge, les inégalités restent significatives mais leur ampleur varie sensiblement d'une cohorte à l'autre et selon l'âge. Ainsi, pour la génération 1941-1955, les inégalités sont particulièrement marquées au début du cycle de vie, puis se réduisent rapidement : la valeur du RII passe ainsi de 8,7 à 15 ans à 1,4 à 25 ans, puis reste sensiblement identique jusqu'à 39 ans. Pour la génération 1956-1970, l'indice reste globalement constant au cours de la vie, à un niveau inférieur à celui observé aux âges jeunes dans la génération précédente, 
mais supérieur à celui observé aux âges adultes dans la génération précédente. Enfin, pour la génération 1971-1985, l'indice reste très élevé (supérieur à 5) à tous les âges et son profil au cours de la vie s'apparente à un profil en U peu marqué, les inégalités étant très accentuées aux âges jeunes (avec des valeurs supérieures à 10) et après 35 ans. Après l'âge de 40 ans, pour les deux cohortes les plus anciennes, les inégalités accusent un léger retrait mais restent significatives jusqu'à l'âge de 52 ans pour la cohorte intermédiaire et jusqu'à l'âge de 42 ans pour la cohorte la plus ancienne.

Chez les femmes, l'indice relatif d'inégalités est largement supérieur à 1 avant 18 ans pour les trois générations considérées. Ceci indique que dans cette tranche d'âge les inégalités sociales ont toujours été de grande ampleur et en défaveur des femmes les moins instruites. Au-delà de cet âge, on assiste dans la génération la plus ancienne à un renversement du sens des inégalités qui se mettent à jouer en défaveur des femmes les plus instruites avec un RII inférieur à 1 . Dans cette génération, à l'approche de la quarantaine, le RII augmente très légèrement, pour se rapprocher de 1 ,en raison des arrêts de consommation relativement plus fréquents parmi les femmes les plus instruites. Dans la génération intermédiaire, les inégalités présentent des valeurs du RII très élevées avant 20 ans et relativement stables après cet

\section{Graphique V}

Indices relatifs d'inégalité (RII, échelle logarithmique) en fonction de l'âge - hommes et femmes des cohortes 1941-1955, 1956-1970 et 1971-1985
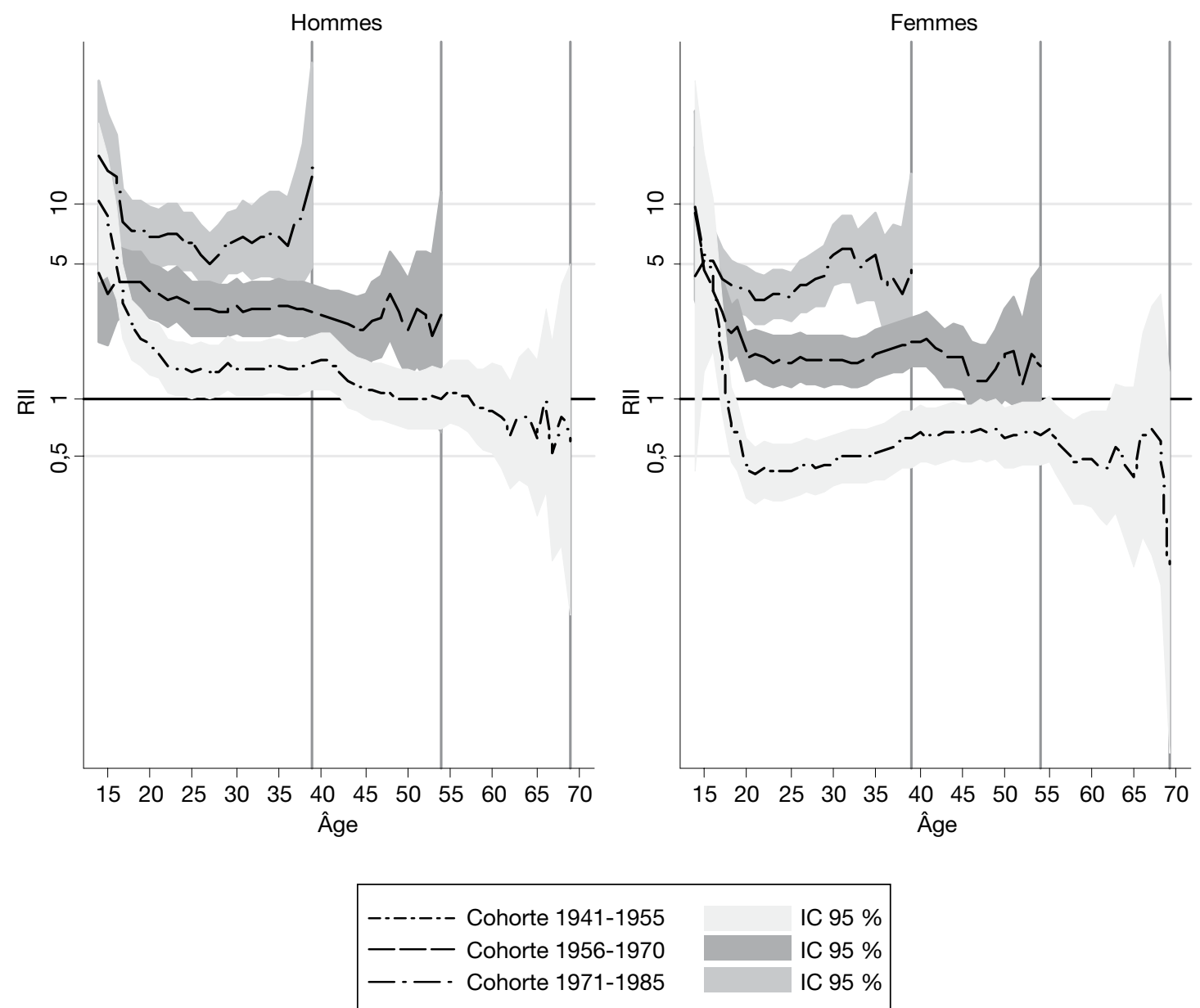

Lecture : les indices relatifs d'inégalité (RII) représentés sur ce graphique sont issus de modélisations logistiques séparées par cohorte et par sexe ; ils sont estimés par interaction entre l'âge rétrospectif et la variable de rang reconstruite en fonction des niveaux de diplôme; ils sont représentés par l'odds ratio associé à cette variable et s'interprètent donc par rapport à 1 .

Dans la génération 1941-1955, les hommes les moins diplômés ont 1,8 fois plus de chances de fumer que les plus diplômés à l'âge de 20 ans (cf. annexe, tableau A pour le détail).

Champ : population française, hommes et femmes âgés de 25 à 69 ans au moment de l'enquête (âgés de 14 à 39 ans pour la cohorte 1971-1985, de 40 à 54 ans pour la cohorte 1956-1970 et de 55 à 69 ans pour la cohorte 1941-1955).

Source : Inpes, Baromètre santé 2010. 
âge, autour de 3 , avec une très légère remontée à partir de 35 ans. Enfin, pour la génération la plus récente, le profil est assez comparable à celui des hommes : le RII reste globalement stable au cours de la vie, mais à un niveau supérieur à celui observé pour l'ensemble des générations précédentes. On retrouve comme pour les hommes un profil «en $\mathrm{U}$ », mais plus étroit (la remontée des inégalités commence à 30 ans contre 36 ans pour les hommes). Les inégalités sont toujours significatives jusqu'à l'âge de 45 ans pour la cohorte intermédiaire alors qu'elles restent en défaveur des femmes les plus instruites jusqu'à l'âge de 62 ans pour la cohorte la plus ancienne.

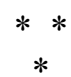

Nos résultats montrent une augmentation importante des inégalités sociales de tabagisme au fil des cohortes chez les hommes et les femmes avec, notamment, une inversion $\mathrm{du}$ gradient social chez les femmes. Cette inversion provient d'une très forte hausse du tabagisme chez les femmes les moins diplômées qui rattrapent ainsi les hommes dont la phase d'inversion a eu lieu auparavant. Ainsi se trouve confirmé le décalage d'une génération entre hommes et femmes dans la diffusion du tabagisme (Legleye et al., 2011b). Chez les hommes d'abord, puis chez les femmes par la suite, l'augmentation des inégalités provient surtout d'une baisse continue du tabagisme des plus diplômés, couplée à sa progression chez les moins diplômés. En outre, l'évolution des inégalités sociales en matière de tabagisme au cours de la vie traduit aussi le décalage hommes-femmes dans la diffusion du tabagisme. Les résultats permettent de souligner l'importance des inégalités sociales face au tabagisme aux âges jeunes, pour toutes les cohortes, aussi bien chez les hommes que chez les femmes. Dans les cohortes les plus anciennes, les inégalités s'amenuisent au cours de la vie jusqu'à s'inverser chez les femmes. Dans la cohorte la plus récente, les inégalités restent à un niveau élevé tout au long du cycle de vie et tendent même à augmenter à nouveau après 25 ans chez les femmes.

L'observation d'une convergence des prévalences tabagiques masculines et féminines confirme les résultats des études sur l'initiation et l'arrêt du tabac (Legleye et al., 2011b). Cette convergence se matérialise par un rapprochement des durées moyennes de tabagisme entre hommes et femmes dans la dernière cohorte, et en particulier pour les hommes et les femmes les plus diplômés. Dans cette dernière catégorie, on observe néanmoins des différences dans les profils tabagiques selon l'âge, les femmes étant davantage enclines à fumer que les hommes avant 25 ans et moins après. Ces différences peuvent sans doute s'expliquer par une tendance des femmes à cesser de fumer à l'approche de la première maternité, et ce, d'autant plus que le niveau d'éducation est élevé (Lelong et al. 2011). L'absence de données datées relatives aux grossesses ne nous permet malheureusement pas de tester cette hypothèse de l'influence sur le tabagisme féminin de l'évolution des calendriers génésiques suivant les cohortes et le diplôme.

Conforme à la théorie de la diffusion des innovations, l'augmentation des inégalités sociales avec les cohortes peut aussi être mise en relation avec les mécanismes reliant le niveau d'éducation et le tabagisme. L'augmentation des inégalités sociales de tabagisme coïncide tout d'abord avec la diffusion de l'information sur la nocivité du tabac. Pour la première cohorte née entre 1941 et 1955 , le fait de fumer dépendait peu des connaissances épidémiologiques, ce qui peut expliquer les faibles niveaux d'inégalité dans cette cohorte. En effet, les informations sur la dangerosité du tabac ne sont réellement sorties du champ académique qu'à partir de 1962 - date du rapport du Royal College of Physicians, Smoking and Health - et ce n'est qu'à partir des années 1970 que ces informations se sont traduites en France par des recommandations de santé publique adoptées sans doute d'abord par les plus éduqués.

Par ailleurs, l'expansion scolaire connue par les cohortes du baby-boom (cohorte 1956-1970) aurait pu conduire à une dévalorisation des diplômes et par là une réduction des inégalités sociales de tabagisme induite par une diminution des effets désincitatifs à fumer de l'éducation. Cependant, malgré une baisse des rendements de l'éducation (Selz-Laurière et Thélot, 2004), le diplôme est devenu un facteur de plus en plus déterminant de l'insertion dans le marché du travail (Guironnet, 2005) et ceci dans un contexte où le risque de chômage et le délai d'attente avant l'obtention d'un emploi stable n'a cessé d'augmenter entre les générations nées dans les années 1940 et celles nées après 1960 (Rouxel et Virely, 2012). Les politiques d'augmentation du prix du tabac des années 2000 auraient pu contrer ces effets en influant 
plus fortement sur la contrainte budgétaire des moins favorisés. Mais cet effet de contrainte budgétaire ne semble pas l'avoir emporté dans la mesure où le tabagisme des chômeurs n'a pas diminué durant cette période alors qu'il baissait plus fortement encore pour les cadres que pour les ouvriers (Peretti-Watel et al., 2009) et ceci d'autant plus que ces effets d'incitation à fumer se cumulent aussi aux effets liés à l'évolution des conditions de vie et des sources de stress (Peretti-Watel et Constance, 2009). Soulignons que les fortes hausses des prix ont été concentrées sur les années 2002-2003, ce qui a entraîné des baisses de la prévalence tabagique, en particulier chez les adolescents (Beck, 2011). En dehors de cette période, les hausses de prix ont été plus modestes, et de ce fait peu propices à un changement de comportement (Legleye et al., 2007). Si ces résultats ne permettent pas de faire la part des différents facteurs, ils suggèrent néanmoins que l'augmentation des inégalités sociales de tabagisme et plus globalement la persistance des inégalités de santé sont liées au maintien des causes sociales de ces inégalités (Mackenbach, 2012; Phelan et al., 2010) de même qu'au profil de plus en plus défavorisé des moins diplômés.

L'utilisation de données rétrospectives sur le tabagisme permet d'avoir le recul nécessaire pour faire une étude historique des inégalités de tabagisme mais elle peut néanmoins souffrir de certains biais. Tout d'abord, la mortalité différentielle des fumeurs peut conduire à une sous-estimation de la prévalence du tabagisme pour les cohortes les plus anciennes. Ce biais lié à la mortalité est minimisé dans notre analyse par la restriction de l'échantillon aux individus de moins de 70 ans comme le suggère une analyse étudiant la validité des données rétrospectives sur le tabagisme (Christopoulou et al., 2011). Le recueil rétrospectif des histoires tabagiques peut aussi souffrir de biais de mémoire et d'erreurs de déclaration. Il a été ainsi démontré que ces biais étaient plus faibles pour les gros fumeurs (Kenkel et al., 2004), mais nous ne pouvons le corriger par manque d'information sur le nombre de cigarettes que les anciens fumeurs avaient l'habitude de fumer. De tels biais peuvent varier selon le niveau d'éducation et ainsi conduire à sous-estimer les inégalités de tabagisme liées au diplôme dans le cas d'une sous-déclaration des moins diplômés. L'absence de données sur le nombre de cigarettes fumées peut aussi nous conduire à sous-estimer le niveau d'inégalité dans la consommation de tabac dans la mesure où le nombre de cigarettes fumées a diminué plus fortement pour les plus favorisés (Godefroy, 2003). D'autre part, cette analyse offre une description de l'évolution des inégalités sans pour autant permettre de distinguer si cette évolution est liée à une évolution dans l'effet du niveau d'éducation ou à une évolution liée à des différences dans la composition des différents niveaux de diplôme. Une telle analyse est complexe car elle nécessiterait une ou plusieurs situations expérimentales, permettant d'estimer l'évolution de l'effet causal de l'éducation sur le tabagisme. Cette relation causale a été étudiée par exemple pour la France au moment de l'expansion scolaire des baby-boomers (Etilé et Jones, 2011).

Malgré ces limites, l'ensemble des résultats va à l'appui d'un ciblage les politiques de prévention du tabagisme selon les groupes sociaux et selon les âges de la vie et suggère en particulier d'axer les efforts de prévention de l'entrée dans le tabagisme quotidien dès les plus jeunes âges dans les milieux les moins favorisés. Il existe à travers le monde des programmes qui ont fait la preuve de leur efficacité en termes de baisse des prévalences tabagiques et de réductions des inégalités sociales de santé : un exemple intéressant est le programme anglais $A$ Stop Smoking In Schools Trial (ASSIST) de prévention par les pairs en milieu scolaire (Hollingworth et al., 2012 ; Audrey et al., 2006 ; Starkey et al., 2005). La mise en œuvre de ce type de programmes de prévention est à encourager et à adapter au contexte français, en regard des initiatives locales déjà déployées (Séquier et al., 2002). La piste du développement du marketing social pour contrer le marketing de l'industrie du tabac dont l'impact sur les jeunes les moins favorisés est particulièrement fort, est également prometteuse (Gallopel-Morvan et al., 2011). 


\section{BIBLIOGRAPHIE}

Audrey S., Holliday J. et Campbell R. (2006), « It's good to talk: adolescent perspectives of an informal, peer-led intervention to reduce smoking ». Social Science and Medicine, vol. 63, n², pp. 320-334.

Balia S. et Jones A. (2008), « Mortality, lifestyle and socio-economic status », Journal of Health Economics, vol. 27, $\mathrm{n}^{\circ} 1$, pp. 1-26.

Balia S. et Jones A. (2011), « Catching the habit: a study of inequality of opportunity in smoking-related mortality ", Journal Of The Royal Statistical Society, Series A, Royal Statistical Society, vol. 174, n 1, pp. 175-194, Janvier.

Beck F. (2011), « Le tabagisme des adolescents : regards croisés de l'épidémiologie et de la sociologie », Médecine/science, $\mathrm{n}^{\circ} 27$, pp. 308-310.

Beck F. et Amsellem-Mainguy Y. (2012), « Les inégalités sociales de santé : vers une prévention mieux ciblée », dans Labadie M. (dir.), Inégalités entre jeunes sur fond de crise, La Documentation française, Paris, pp. 124-136.

Beck F., Gautier A., Guignard R. et Richard J.-B. (2011), «Une méthode de prise en compte du dégroupage total dans le plan de sondage des enquêtes téléphoniques auprès des ménages ", dans Tremblay M.-E., Lavallée P. et El Hadj Tirari M. (dir.), Pratiques et Méthodes de sondage, Collection Sciences Sup, Dunod, Paris, pp. 310-314.

Braakmann N. (2011), «The causal relationship between education, health and health related behaviour: Evidence from a natural experiment in England », Journal of Health Economics, vol. 30, $\mathrm{n}^{\circ} 4$, pp. 753-763.

Bricard D. et Jusot F. (2012), « Milieu d'origine, situation sociale et parcours tabagique en France », Économie Publique, n 28-29, pp. 169-195.

Christopoulou R., Han J., Jaber A. et Lillard D. R. (2011), « Dying for a smoke: How much does differential mortality of smokers affect estimated lifecourse smoking prevalence? $»$, Preventive medicine, vol. 52, $\mathrm{n}^{\circ}$ 1, pp. 66-70.

Cutler D. M. et Lleras-Muney A. (2010), «Understanding differences in health behaviors by education ». Journal of Health Economics, vol. $29, \mathrm{n}^{\circ} 1$, pp. 1-28.
Cutler D.M., Lange F., Meara E., RichardsShubik S. et Ruhm C.J. (2011), « Rising educational gradients in mortality: The role of behavioral risk factors ", Journal of Health Economics, vol. $30, \mathrm{n}^{\circ} 6$, pp. 1174-1187.

Etilé F. (2007), «Modes de vie et santé des jeunes », dans Cohen D. (éd.) : Une jeunesse diffcile. Portrait économique et social de la jeunesse française, Éditions Rue d'Ulm/Presses de l'École Normale Supérieure, Paris.

Etilé F. et Jones A. (2011), « Schooling and smoking among the baby-boomers: an evaluation of the impact of educational expansion in France », Journal of Health Economics; vol. 30, $\mathrm{n}^{\circ} 4$, pp. 811-831.

Federico B., Costa G. et Kunst A. E. (2007), «Educational inequalities in initiation, cessation, and prevalence of smoking among 3 Italian birth cohorts », American journal of public health, vol. $97, \mathrm{n}^{\circ} 5$, pp. 838-845.

Fernandez E., Garcia M., Schiaffino A., Borras J. M., Nebot M. et Segura A. (2001), « Smoking initiation and cessation by gender and educational level in Catalonia », Espagne, Preventive medicine, vol. $32, \mathrm{n}^{\circ} 3$, pp. 218-223.

Gallopel-Morvan K., Gabriel P., Le Gall-Ely M., Rieunier S. et Urien B. (2011), "The use of visual warnings in social marketing: the case of tobacco », Journal of Business Research, vol. 64, $\mathrm{n}^{\circ} 1$, pp. 7-11.

Godefroy R. (2003), "Les taxes sur le tabac sont-elles régressives? », Économie publique, vol. $2, \mathrm{n}^{\circ} 13$, pp. 3-28.

Guironnet J.-P. (2006), « La suréducation en France : vers une dévalorisation des diplômes du supérieur?». Économie appliquée, vol. 59, n 1 , pp. 93-120.

Hayes L. J. (2002), « Sampling variability of the Kunst-Mackenbach relative index of inequality », Journal of Epidemiology and Community health, $\mathrm{n}^{\circ} 56$, pp. 762-765.

Hollingworth W., Cohen D., Hawkins J., Hughes R.A., Moore L.A., Holliday J.C., Audrey S., Starkey F. et Campbell R. (2012), «Reducing smoking in adolescents: cost-effectiveness results from the cluster randomized 
ASSIST (A Stop Smoking In Schools Trial) », Nicotine TobRes., vol. 14, n ${ }^{\circ}$, pp. 161-168.

Jefferis B. J., Power C., Graham H. et Manor O. (2004), «Changing social gradients in cigarette smoking and cessation over two decades of adult follow-up in a British birth cohort », Journal of Public Health, vol. 26, $\mathrm{n}^{\circ}$ 1, pp. 13-18.

Kenkel D. S., Lillard D. R. et Mathios A. D. (2004), «Accounting for misclassification error in retrospective smoking data », Health Economics, vol. $13, \mathrm{n}^{\circ} 10$, pp. 1031-1044.

Khaw K.T., Wareham N., Bingham S., Welch A., Luben R. et Day N. (2008), « Combined impact of health behaviours and mortality in men and women: the EPIC-Norfolk prospective population study », PLoS Medicine; vol. 5, $\mathrm{n}^{\circ} 1$, pp. 39-47.

Legleye S., Beck F. et Peretti-Watel P. (2007), «Tabagisme en France: Impact des hausses des prix: transitoire? ", La Revue du praticien, Médecine générale, $\mathrm{n}^{\circ}$ 756-757, pp. 75-78.

Legleye S., Janssen E., Beck F., Chau N. et Khlat M. (2011a), « Social gradient in initiation and transition to daily use of tobacco and cannabis during adolescence: a retrospective cohort study », Addiction, vol. 106, n 8, pp. 1520-1531.

Legleye S., Khlat M., Beck F. et Peretti-Watel P. (2011b), «Widening inequalities in smoking initiation and cessation patterns: A cohort and gender analysis in France », Drug and Alcohol Dependence, vol. 117, $\mathrm{n}^{\circ} 2-3$, pp. 233-241.

Lelong N., Blondel B. et Kaminski M. (2011), «Évolution de la consommation de tabac des femmes pendant la grossesse en France de 1972 à 2003 », Journal de gynécologie obstétrique et biologie de la reproduction, vol. 40, $\mathrm{n}^{\circ} 1$, pp. 42-49.

Lopez A.D., Collishaw N.E. et Piha T. (1994), «A descriptive model of the cigarette epidemic in developed countries », Tobacco Control, vol. 3, $\mathrm{n}^{\circ} 3$, pp. 242-247.

Mackenbach J.P. et Kunst A. (1997), « Measuring the magnitude of socio-economic inequalities in health: An overview of available measures illustrated with two examples from Europe », Social Science and Medicine, vol. 44, $\mathrm{n}^{\circ}$ 6, pp. 757-771.

Mackenbach J.P. (2011), « What would happen to health inequalities if smoking were eliminated », British Medical Journal; n 342, pp. 1-2.
Mackenbach J.P., Stirbu I., Roskam A.J., Schaap M.M., Menvielle G., Leinsalu M., Kunst A.E., European Union Working Group on Socioeconomic Inequalities in Health. et al., (2008), « Socioeconomic inequalities in health in 22 European countries ", The New England Journal of Medicine, vol. 358, $\mathrm{n}^{\circ} 23$, pp. 2468-2481.

Mackenbach J.P. (2012), « The persistence of health inequalities in modern welfare states: the explanation of a paradox », Social Science and Medicine, vol. 75, $\mathrm{n}^{\circ}$ 4, pp. 761-769.

Maralani V. (2013), « Educational inequalities in smoking: the role of initiation versus quitting », Social Science and Medicine, vol. 84, pp. 129-137.

Nourrisson D. (1999), Histoire sociale du tabac, Editions Christian, Paris.

OECD (1999), Classifying Educational Programmes Manual for ISCED-97 Implementation in OECD Countries, OECD, Paris.

Pampel F. C., Krueger P. M. et Denney J. T. (2010), " Socioeconomic disparities in health behaviors », Annual review of sociology, $\mathrm{n}^{\circ} 36, \mathrm{pp} .349-370$.

Peretti-Watel P., Seror V., Constance J. et Beck F., (2008), « Poverty as a smoking trap », International Journal of Drug Policy, special issue on « risk environments », vol. 20, n 3 , pp. 230-236.

Peretti-Watel P., Constance J., Seror V. et Beck F. (2009), «Cigarettes and social differentiation in France: is tobacco use increasingly concentrated among the poor?», Addiction, vol. 104, $\mathrm{n}^{\circ} 10$, pp. $1718-1728$.

Peretti-Watel P. et Constance J. (2009), « It's all we got left ». Why poor smokers are less sensitive to cigarette price increases, International journal of environmental research and public health, vol. $6, \mathrm{n}^{\circ} 2$, pp. 608-621.

Phelan J. C., Link B. G. et Tehranifar P. (2010), «Social conditions as fundamental causes of health inequalities theory, evidence, and policy implications ",, Journal of Health and Social Behavior, vol. 51, $\mathrm{n}^{\circ} 1$, supplément, pp. 28-40.

Pison G. (2010), « France 2009 : l'âge moyen à la maternité atteint 30 ans ", Population et sociétés, $\mathrm{n}^{\circ} 465$, pp. 1-4.

Rogers E.M. et Shoemaker F.F., (1971), Communication of Innovations: A Cross-Cultural Approach, 2e éd., Free Press, New York. 
Rouxel C. et Virely B. (2012), « Les transformations des parcours d'emploi et de travail au fil des générations » dans Emploi et Salaires, Insee Références, 2012.

Schiaffino A., Fernandez E., Borrell C., Salto E., Garcia M. et Borras J. M. (2003), «Gender and educational differences in smoking initiation rates in Spain from 1948 to 1992 », The European Journal of Public Health, vol. 13, $\mathrm{n}^{\circ} 1$, pp. 56-60.

Selz-Laurière M. et Thélot C. (2004), «The returns to education and experience: Trends in France over the last thirty-five years », Ined, Population (édition anglaise), vol. 59, $\mathrm{n}^{\circ}$ 1, pp. 9-48.

Séquier A., Stoebner A., Gourgou S., Lence J.J., Bonifaci C. et Sancho-Garnier H. (2002), « Educational methods in the prevention of smoking, among students of the Department of Herault, France », Salud Publica Mex. ; vol. 44, $\mathrm{n}^{\circ} 1$, pp. 93-100.

Starkey F., Moore L., Campbell R., Sidaway M. et Bloor M. (2005), « ASSIST. Rationale, design and conduct of a comprehensive evaluation of a school-based peer-led anti-smoking intervention in the UK: the ASSIST cluster randomised trial [ISRCTN55572965] », BMC Public Health, vol. $22, \mathrm{n}^{\circ} 5$, pp. 43-53.

Stringhini S., Sabia S., Shipley M., Brunner E., Nabi H., Kivimaki M. et Singh-Manoux A. (2010), «Association of Socioeconomic Position with Health Behaviors and Mortality », Journal of the American Medical Association, vol. 303, $\mathrm{n}^{\circ} 12$, pp. 1159-1166.

Thun M., Peto R., Boreham J. et Lopez A. D. (2012), " Stages of the cigarette epidemic on entering its second century », Tobacco control, vol. 21, $\mathrm{n}^{\circ} 2$, pp. 96-101.

Tubeuf S., Jusot F. et Bricard D. (2012), «Mediating role of education and lifestyles in the relationship between early-life conditions and health: Evidence from the 1958 British cohort », Health Economics, vol. 21, n ${ }^{\circ}$, pp. 129-150.

Wagstaff A., Paci P. et Van Doorslaer E. (1991), " On the measurement of inequalities in health", Social Science and Medicine, vol. 33, $\mathrm{n}^{\circ}$ 5, pp. 545-557.

Waldron I. (1991), « Patterns and causes of gender differences in smoking », Social Science and Medicine, vol. 32, n 9, pp. 989-1005. 
Tableau A

Régressions logistiques de la probabilité d'être fumeur selon la cohorte de naissance chez les hommes

\begin{tabular}{|c|c|c|c|c|c|c|}
\hline \multirow[t]{2}{*}{ Cohorte } & \multicolumn{2}{|c|}{ 1941-1955 } & \multicolumn{2}{|c|}{ 1956-1970 } & \multicolumn{2}{|c|}{ 1971-1985 } \\
\hline & O.R. & $\mathrm{p}$-value & O.R. & $\mathrm{p}$-value & O.R. & $\mathrm{p}$-value \\
\hline Annais & 0,99 & 0,41 & 1,00 & 0,75 & 0,99 & 0,21 \\
\hline \multicolumn{7}{|c|}{ Rang * âge rétrospectif a } \\
\hline 14 & 10,32 & 0,00 & 4,37 & 0,00 & 17,80 & 0,00 \\
\hline 15 & 8,70 & 0,00 & 3,41 & 0,00 & 14,68 & 0,00 \\
\hline 16 & 5,58 & 0,00 & 3,97 & 0,00 & 13,90 & 0,00 \\
\hline 17 & 3,13 & 0,00 & 3,95 & 0,00 & 8,15 & 0,00 \\
\hline 18 & 2,25 & 0,00 & 4,02 & 0,00 & 7,40 & 0,00 \\
\hline 19 & 2,04 & 0,00 & 4,05 & 0,00 & 7,36 & 0,00 \\
\hline 20 & 1,83 & 0,00 & 3,57 & 0,00 & 6,89 & 0,00 \\
\hline 21 & 1,73 & 0,00 & 3,41 & 0,00 & 6,73 & 0,00 \\
\hline 22 & 1,46 & 0,02 & 3,25 & 0,00 & 7,08 & 0,00 \\
\hline 23 & 1,42 & 0,03 & 3,39 & 0,00 & 7,09 & 0,00 \\
\hline 24 & 1,41 & 0,04 & 3,18 & 0,00 & 6,43 & 0,00 \\
\hline 25 & 1,35 & 0,07 & 2,87 & 0,00 & 6,39 & 0,00 \\
\hline 26 & 1,39 & 0,04 & 2,90 & 0,00 & 5,54 & 0,00 \\
\hline 27 & 1,35 & 0,06 & 2,88 & 0,00 & 4,97 & 0,00 \\
\hline 28 & 1,38 & 0,05 & 2,83 & 0,00 & 5,45 & 0,00 \\
\hline 29 & 1,51 & 0,01 & 2,82 & 0,00 & 6,31 & 0,00 \\
\hline 30 & 1,39 & 0,04 & 2,96 & 0,00 & 6,48 & 0,00 \\
\hline 31 & 1,40 & 0,04 & 2,83 & 0,00 & 6,87 & 0,00 \\
\hline 32 & 1,40 & 0,04 & 2,85 & 0,00 & 6,40 & 0,00 \\
\hline 33 & 1,42 & 0,03 & 2,90 & 0,00 & 6,75 & 0,00 \\
\hline 34 & 1,45 & 0,02 & 2,91 & 0,00 & 7,09 & 0,00 \\
\hline 35 & 1,44 & 0,03 & 2,96 & 0,00 & 6,93 & 0,00 \\
\hline 36 & 1,41 & 0,04 & 2,93 & 0,00 & 6,11 & 0,00 \\
\hline 37 & 1,40 & 0,04 & 2,92 & 0,00 & 8,01 & 0,00 \\
\hline 38 & 1,45 & 0,03 & 2,88 & 0,00 & 9,14 & 0,00 \\
\hline 39 & 1,53 & 0,01 & 2,81 & 0,00 & 16,04 & 0,00 \\
\hline 40 & 1,54 & 0,01 & 2,67 & 0,00 & & \\
\hline 41 & 1,54 & 0,01 & 2,56 & 0,00 & & \\
\hline 42 & 1,40 & 0,05 & 2,48 & 0,00 & & \\
\hline 43 & 1,24 & 0,20 & 2,38 & 0,00 & & \\
\hline 44 & 1,17 & 0,36 & 2,27 & 0,00 & & \\
\hline 45 & 1,13 & 0,49 & 2,24 & 0,00 & & \\
\hline 46 & 1,08 & 0,65 & 2,49 & 0,00 & & \\
\hline 47 & 1,08 & 0,67 & 2,62 & 0,00 & & \\
\hline 48 & 1,05 & 0,78 & 3,40 & 0,00 & & \\
\hline 49 & 0,99 & 0,97 & 2,77 & 0,00 & & \\
\hline 50 & 0,98 & 0,92 & 2,26 & 0,01 & & \\
\hline 51 & 0,97 & 0,88 & 2,86 & 0,00 & & \\
\hline 52 & 0,98 & 0,93 & 2,71 & 0,01 & & \\
\hline 53 & 1,01 & 0,95 & 2,09 & 0,14 & & \\
\hline 54 & 0,98 & 0,90 & 2,79 & 0,16 & & \\
\hline 55 & 1,07 & 0,72 & & & & $\rightarrow$ \\
\hline
\end{tabular}


Tableau A (suite)

\begin{tabular}{|c|c|c|c|c|c|c|}
\hline \multirow[t]{2}{*}{ Cohorte } & \multicolumn{2}{|c|}{$1941-1955$} & \multicolumn{2}{|c|}{$1956-1970$} & \multicolumn{2}{|c|}{ 1971-1985 } \\
\hline & O.R. & $\mathrm{p}$-value & O.R. & $p$-value & O.R. & $p$-value \\
\hline \multicolumn{7}{|c|}{ Rang * âge rétrospectif a } \\
\hline 56 & 1,04 & 0,84 & & & & \\
\hline 57 & 1,02 & 0,93 & & & & \\
\hline 58 & 0,89 & 0,61 & & & & \\
\hline 59 & 0,87 & 0,57 & & & & \\
\hline 60 & 0,87 & 0,61 & & & & \\
\hline 61 & 0,79 & 0,44 & & & & \\
\hline 62 & 0,64 & 0,18 & & & & \\
\hline 63 & 0,79 & 0,52 & & & & \\
\hline 64 & 0,79 & 0,58 & & & & \\
\hline 65 & 0,62 & 0,30 & & & & \\
\hline 66 & 0,97 & 0,95 & & & & \\
\hline 67 & 0,52 & 0,33 & & & & \\
\hline 68 & 0,81 & 0,79 & & & & \\
\hline 69 & 0,60 & 0,63 & & & & \\
\hline \multicolumn{7}{|c|}{ Âge rétrospectif a } \\
\hline 14 & 0,02 & 0,00 & 0,04 & 0,00 & 0,03 & 0,00 \\
\hline 15 & 0,03 & 0,00 & 0,09 & 0,00 & 0,07 & 0,00 \\
\hline 16 & 0,07 & 0,00 & 0,19 & 0,00 & 0,16 & 0,00 \\
\hline 17 & 0,16 & 0,00 & 0,31 & 0,00 & 0,36 & 0,00 \\
\hline 18 & 0,40 & 0,00 & 0,56 & 0,00 & 0,63 & 0,00 \\
\hline 19 & 0,51 & 0,00 & 0,64 & 0,00 & 0,73 & 0,00 \\
\hline 21 & 1,13 & 0,00 & 1,08 & 0,00 & 1,09 & 0,00 \\
\hline 22 & 1,40 & 0,00 & 1,17 & 0,00 & 1,13 & 0,00 \\
\hline 23 & 1,45 & 0,00 & 1,16 & 0,00 & 1,13 & 0,01 \\
\hline 24 & 1,47 & 0,00 & 1,21 & 0,00 & 1,21 & 0,00 \\
\hline 25 & 1,63 & 0,00 & 1,37 & 0,00 & 1,25 & 0,00 \\
\hline 26 & 1,57 & 0,00 & 1,34 & 0,00 & 1,32 & 0,00 \\
\hline 27 & 1,56 & 0,00 & 1,32 & 0,00 & 1,35 & 0,00 \\
\hline 28 & 1,49 & 0,00 & 1,32 & 0,00 & 1,29 & 0,00 \\
\hline 29 & 1,36 & 0,00 & 1,28 & 0,00 & 1,16 & 0,12 \\
\hline 30 & 1,47 & 0,00 & 1,24 & 0,00 & 1,11 & 0,32 \\
\hline 31 & 1,40 & 0,00 & 1,23 & 0,01 & 1,01 & 0,94 \\
\hline 32 & 1,34 & 0,00 & 1,19 & 0,02 & 0,99 & 0,94 \\
\hline 33 & 1,26 & 0,01 & 1,12 & 0,13 & 0,91 & 0,50 \\
\hline 34 & 1,21 & 0,05 & 1,05 & 0,52 & 0,88 & 0,39 \\
\hline 35 & 1,16 & 0,13 & 1,01 & 0,91 & 0,81 & 0,21 \\
\hline 36 & 1,12 & 0,23 & 0,99 & 0,90 & 0,89 & 0,53 \\
\hline 37 & 1,09 & 0,39 & 0,95 & 0,58 & 0,80 & 0,27 \\
\hline 38 & 1,02 & 0,82 & 0,92 & 0,34 & 0,69 & 0,14 \\
\hline 39 & 0,93 & 0,48 & 0,89 & 0,19 & 0,55 & 0,10 \\
\hline 40 & 0,88 & 0,19 & 0,88 & 0,14 & & \\
\hline 41 & 0,84 & 0,09 & 0,86 & 0,11 & & \\
\hline 42 & 0,84 & 0,09 & 0,83 & 0,07 & & \\
\hline 43 & 0,85 & 0,11 & 0,81 & 0,06 & & \\
\hline 44 & 0,84 & 0,09 & 0,80 & 0,06 & & \\
\hline 45 & 0,81 & 0,05 & 0,77 & 0,04 & & \\
\hline 46 & 0,79 & 0,02 & 0,67 & 0,00 & & -3 \\
\hline
\end{tabular}


Tableau A (suite)

\begin{tabular}{|c|c|c|c|c|c|c|}
\hline \multirow[t]{2}{*}{ Cohorte } & \multicolumn{2}{|c|}{ 1941-1955 } & \multicolumn{2}{|c|}{ 1956-1970 } & \multicolumn{2}{|c|}{ 1971-1985 } \\
\hline & O.R. & $\mathrm{p}$-value & O.R. & $\mathrm{p}$-value & O.R. & $\mathrm{p}$-value \\
\hline \multicolumn{7}{|c|}{ Âge rétrospectif a } \\
\hline 47 & 0,76 & 0,01 & 0,67 & 0,01 & & \\
\hline 48 & 0,73 & 0,00 & 0,55 & 0,00 & & \\
\hline 49 & 0,71 & 0,00 & 0,58 & 0,00 & & \\
\hline 50 & 0,68 & 0,00 & 0,60 & 0,00 & & \\
\hline 51 & 0,64 & 0,00 & 0,52 & 0,00 & & \\
\hline 52 & 0,61 & 0,00 & 0,48 & 0,00 & & \\
\hline 53 & 0,56 & 0,00 & 0,58 & 0,06 & & \\
\hline 54 & 0,54 & 0,00 & 0,43 & 0,05 & & \\
\hline 55 & 0,49 & 0,00 & & & & \\
\hline 56 & 0,46 & 0,00 & & & & \\
\hline 57 & 0,44 & 0,00 & & & & \\
\hline 58 & 0,44 & 0,00 & & & & \\
\hline 59 & 0,41 & 0,00 & & & & \\
\hline 60 & 0,37 & 0,00 & & & & \\
\hline 61 & 0,37 & 0,00 & & & & \\
\hline 62 & 0,39 & 0,00 & & & & \\
\hline 63 & 0,33 & 0,00 & & & & \\
\hline 64 & 0,32 & 0,00 & & & & \\
\hline 65 & 0,37 & 0,00 & & & & \\
\hline 66 & 0,29 & 0,00 & & & & \\
\hline 67 & 0,33 & 0,00 & & & & \\
\hline 68 & 0,26 & 0,00 & & & & \\
\hline 69 & 0,30 & 0,03 & & & & \\
\hline Pseudo $\mathrm{R}^{2}$ & \multicolumn{2}{|c|}{0,0741} & \multicolumn{2}{|c|}{0,0662} & \multicolumn{2}{|c|}{0,1074} \\
\hline Obs. & \multicolumn{2}{|c|}{134518} & \multicolumn{2}{|c|}{110328} & \multicolumn{2}{|c|}{63483} \\
\hline Ind. & \multicolumn{2}{|c|}{2790} & \multicolumn{2}{|c|}{3286} & \multicolumn{2}{|c|}{3249} \\
\hline
\end{tabular}

Lecture : dans la génération 1941-1955, les hommes les moins diplômés ont 1,8 fois plus de chances de fumer que les plus diplômés à l'âge de 20 ans.

Champ : population française, hommes âges de 25 à 69 ans au moment de l'enquête (âgés de 14 à 39 ans pour la cohorte 1971-1985, de 40 à 54 ans pour la cohorte 1956-1970 et de 55 à 65 ans pour la cohorte 1941-1955).

Source : Inpes, Baromètre santé 2010. 
Tableau B

Régressions logistiques de la probabilité d'être fumeur selon la cohorte de naissance chez les femmes

\begin{tabular}{|c|c|c|c|c|c|c|}
\hline \multirow[t]{2}{*}{ Cohorte } & \multicolumn{2}{|c|}{ 1941-1955 } & \multicolumn{2}{|c|}{$1956-1970$} & \multicolumn{2}{|c|}{ 1971-1985 } \\
\hline & O.R. & $\mathrm{p}$-value & O.R. & $p$-value & O.R. & $\mathrm{p}$-value \\
\hline Annais & 0,92 & 0,00 & 0,98 & 0,04 & 0,99 & 0,35 \\
\hline \multicolumn{7}{|c|}{ Rang * âge rétrospectif a } \\
\hline 14 & 4,26 & 0,22 & 9,81 & 0,00 & 9,08 & 0,00 \\
\hline 15 & 5,10 & 0,01 & 4,55 & 0,00 & 5,35 & 0,00 \\
\hline 16 & 4,36 & 0,00 & 3,59 & 0,00 & 5,14 & 0,00 \\
\hline 17 & 1,61 & 0,17 & 2,79 & 0,00 & 4,12 & 0,00 \\
\hline 18 & 0,73 & 0,17 & 2,18 & 0,00 & 3,78 & 0,00 \\
\hline 19 & 0,63 & 0,03 & 2,29 & 0,00 & 3,72 & 0,00 \\
\hline 20 & 0,43 & 0,00 & 1,62 & 0,00 & 3,55 & 0,00 \\
\hline 21 & 0,40 & 0,00 & 1,69 & 0,00 & 3,25 & 0,00 \\
\hline 22 & 0,43 & 0,00 & 1,60 & 0,00 & 3,24 & 0,00 \\
\hline 23 & 0,41 & 0,00 & 1,53 & 0,00 & 3,41 & 0,00 \\
\hline 24 & 0,42 & 0,00 & 1,56 & 0,00 & 3,41 & 0,00 \\
\hline 25 & 0,41 & 0,00 & 1,53 & 0,00 & 3,35 & 0,00 \\
\hline 26 & 0,44 & 0,00 & 1,61 & 0,00 & 3,76 & 0,00 \\
\hline 27 & 0,44 & 0,00 & 1,56 & 0,00 & 3,88 & 0,00 \\
\hline 28 & 0,44 & 0,00 & 1,58 & 0,00 & 4,13 & 0,00 \\
\hline 29 & 0,44 & 0,00 & 1,55 & 0,00 & 4,24 & 0,00 \\
\hline 30 & 0,47 & 0,00 & 1,55 & 0,00 & 5,48 & 0,00 \\
\hline 31 & 0,49 & 0,00 & 1,56 & 0,00 & 5,90 & 0,00 \\
\hline 32 & 0,51 & 0,00 & 1,51 & 0,01 & 5,90 & 0,00 \\
\hline 33 & 0,50 & 0,00 & 1,51 & 0,01 & 4,77 & 0,00 \\
\hline 34 & 0,50 & 0,00 & 1,59 & 0,00 & 5,06 & 0,00 \\
\hline 35 & 0,51 & 0,00 & 1,67 & 0,00 & 5,46 & 0,00 \\
\hline 36 & 0,53 & 0,00 & 1,75 & 0,00 & 3,83 & 0,00 \\
\hline 37 & 0,56 & 0,00 & 1,81 & 0,00 & 4,15 & 0,00 \\
\hline 38 & 0,61 & 0,00 & 1,85 & 0,00 & 3,41 & 0,00 \\
\hline 39 & 0,61 & 0,00 & 1,94 & 0,00 & 4,51 & 0,01 \\
\hline 40 & 0,65 & 0,01 & 1,97 & 0,00 & & \\
\hline 41 & 0,65 & 0,01 & 2,03 & 0,00 & & \\
\hline 42 & 0,63 & 0,01 & 1,82 & 0,00 & & \\
\hline 43 & 0,66 & 0,02 & 1,62 & 0,01 & & \\
\hline 44 & 0,67 & 0,02 & 1,66 & 0,01 & & \\
\hline 45 & 0,66 & 0,02 & 1,60 & 0,02 & & \\
\hline 46 & 0,66 & 0,02 & 1,28 & 0,23 & & \\
\hline 47 & 0,69 & 0,04 & 1,21 & 0,38 & & \\
\hline 48 & 0,67 & 0,03 & 1,20 & 0,44 & & \\
\hline 49 & 0,68 & 0,04 & 1,39 & 0,19 & & \\
\hline 50 & 0,62 & 0,01 & 1,66 & 0,07 & & \\
\hline 51 & 0,63 & 0,02 & 1,74 & 0,09 & & \\
\hline 52 & 0,65 & 0,03 & 1,19 & 0,64 & & \\
\hline 53 & 0,66 & 0,03 & 1,69 & 0,25 & & \\
\hline 54 & 0,65 & 0,03 & 1,48 & 0,51 & & \\
\hline 55 & 0,68 & 0,06 & & & & \\
\hline 56 & 0,61 & 0,02 & & & & \\
\hline 57 & 0,53 & 0,01 & & & & \\
\hline 58 & 0,46 & 0,00 & & & & $\rightarrow$ \\
\hline
\end{tabular}


Tableau B (suite)

\begin{tabular}{|c|c|c|c|c|c|c|}
\hline \multirow[t]{2}{*}{ Cohorte } & \multicolumn{2}{|c|}{$1941-1955$} & \multicolumn{2}{|c|}{$1956-1970$} & \multicolumn{2}{|c|}{$1971-1985$} \\
\hline & O.R. & $\mathrm{p}$-value & O.R. & $\mathrm{p}$-value & O.R. & $\mathrm{p}$-value \\
\hline \multicolumn{7}{|c|}{ Rang * âge rétrospectif a } \\
\hline 59 & 0,48 & 0,01 & & & & \\
\hline 60 & 0,48 & 0,01 & & & & \\
\hline 61 & 0,45 & 0,02 & & & & \\
\hline 62 & 0,43 & 0,02 & & & & \\
\hline 63 & 0,55 & 0,13 & & & & \\
\hline 64 & 0,46 & 0,09 & & & & \\
\hline 65 & 0,39 & 0,08 & & & & \\
\hline 66 & 0,63 & 0,46 & & & & \\
\hline 67 & 0,68 & 0,61 & & & & \\
\hline 68 & 0,60 & 0,57 & & & & \\
\hline 69 & 0,14 & 0,09 & & & & \\
\hline \multicolumn{7}{|c|}{ Âge rétrospectif a } \\
\hline 14 & 0,01 & 0,00 & 0,01 & 0,00 & 0,04 & 0,00 \\
\hline 15 & 0,02 & 0,00 & 0,06 & 0,00 & 0,12 & 0,00 \\
\hline 16 & 0,04 & 0,00 & 0,13 & 0,00 & 0,23 & 0,00 \\
\hline 17 & 0,13 & 0,00 & 0,23 & 0,00 & 0,40 & 0,00 \\
\hline 18 & 0,42 & 0,00 & 0,52 & 0,00 & 0,69 & 0,00 \\
\hline 19 & 0,53 & 0,00 & 0,56 & 0,00 & 0,76 & 0,00 \\
\hline 21 & 1,12 & 0,00 & 1,02 & 0,38 & 1,07 & 0,01 \\
\hline 22 & 1,16 & 0,00 & 1,09 & 0,02 & 1,10 & 0,00 \\
\hline 23 & 1,22 & 0,00 & 1,12 & 0,01 & 1,07 & 0,09 \\
\hline 24 & 1,22 & 0,00 & 1,11 & 0,03 & 1,04 & 0,41 \\
\hline 25 & 1,43 & 0,00 & 1,19 & 0,00 & 1,03 & 0,53 \\
\hline 26 & 1,39 & 0,00 & 1,15 & 0,02 & 0,93 & 0,19 \\
\hline 27 & 1,39 & 0,00 & 1,14 & 0,02 & 0,90 & 0,12 \\
\hline 28 & 1,40 & 0,00 & 1,11 & 0,08 & 0,85 & 0,03 \\
\hline 29 & 1,38 & 0,00 & 1,09 & 0,18 & 0,81 & 0,01 \\
\hline 30 & 1,50 & 0,00 & 1,11 & 0,14 & 0,68 & 0,00 \\
\hline 31 & 1,45 & 0,00 & 1,07 & 0,32 & 0,65 & 0,00 \\
\hline 32 & 1,40 & 0,00 & 1,05 & 0,49 & 0,61 & 0,00 \\
\hline 33 & 1,37 & 0,00 & 1,03 & 0,72 & 0,65 & 0,00 \\
\hline 34 & 1,36 & 0,00 & 0,97 & 0,67 & 0,63 & 0,00 \\
\hline 35 & 1,36 & 0,00 & 0,92 & 0,27 & 0,57 & 0,00 \\
\hline 36 & 1,29 & 0,00 & 0,87 & 0,09 & 0,67 & 0,02 \\
\hline 37 & 1,19 & 0,06 & 0,84 & 0,03 & 0,62 & 0,02 \\
\hline 38 & 1,12 & 0,23 & 0,81 & 0,01 & 0,64 & 0,06 \\
\hline 39 & 1,09 & 0,37 & 0,76 & 0,00 & 0,52 & 0,06 \\
\hline 40 & 1,07 & 0,50 & 0,73 & 0,00 & & \\
\hline 41 & 1,05 & 0,62 & 0,72 & 0,00 & & \\
\hline 42 & 1,04 & 0,70 & 0,73 & 0,00 & & \\
\hline 43 & 0,98 & 0,86 & 0,75 & 0,00 & & \\
\hline 44 & 0,94 & 0,59 & 0,73 & 0,00 & & \\
\hline 45 & 0,94 & 0,57 & 0,72 & 0,00 & & \\
\hline 46 & 0,91 & 0,40 & 0,78 & 0,05 & & \\
\hline 47 & 0,85 & 0,13 & 0,79 & 0,07 & & \\
\hline 48 & 0,83 & 0,09 & 0,80 & 0,12 & & \\
\hline 49 & 0,79 & 0,04 & 0,76 & 0,06 & & \\
\hline
\end{tabular}


Tableau B (suite)

\begin{tabular}{|c|c|c|c|c|c|c|}
\hline \multirow[t]{2}{*}{ Cohorte } & \multicolumn{2}{|c|}{ 1941-1955 } & \multicolumn{2}{|c|}{ 1956-1970 } & \multicolumn{2}{|c|}{$1971-1985$} \\
\hline & O.R. & $p$-value & O.R. & $p$-value & O.R. & $p$-value \\
\hline \multicolumn{7}{|c|}{ Âge rétrospectif a } \\
\hline 50 & 0,80 & 0,05 & 0,68 & 0,02 & & \\
\hline 51 & 0,77 & 0,02 & 0,66 & 0,03 & & \\
\hline 52 & 0,73 & 0,01 & 0,80 & 0,30 & & \\
\hline 53 & 0,71 & 0,00 & 0,69 & 0,15 & & \\
\hline 54 & 0,70 & 0,00 & 0,65 & 0,22 & & \\
\hline 55 & 0,65 & 0,00 & & & & \\
\hline 56 & 0,68 & 0,00 & & & & \\
\hline 57 & 0,69 & 0,01 & & & & \\
\hline 58 & 0,69 & 0,01 & & & & \\
\hline 59 & 0,65 & 0,01 & & & & \\
\hline 60 & 0,64 & 0,01 & & & & \\
\hline 61 & 0,63 & 0,01 & & & & \\
\hline 62 & 0,64 & 0,02 & & & & \\
\hline 63 & 0,57 & 0,01 & & & & \\
\hline 64 & 0,60 & 0,03 & & & & \\
\hline 65 & 0,61 & 0,07 & & & & \\
\hline 66 & 0,55 & 0,07 & & & & \\
\hline 67 & 0,53 & 0,11 & & & & \\
\hline 68 & 0,69 & 0,41 & & & & \\
\hline 69 & 1,46 & 0,50 & & & & \\
\hline Pseudo- $\mathrm{R}^{2}$ & \multicolumn{2}{|c|}{0,0540} & \multicolumn{2}{|c|}{0,0460} & \multicolumn{2}{|c|}{0,0722} \\
\hline Obs. & \multicolumn{2}{|c|}{185547} & \multicolumn{2}{|c|}{132906} & \multicolumn{2}{|c|}{74836} \\
\hline Ind. & \multicolumn{2}{|c|}{3848} & \multicolumn{2}{|c|}{3925} & \multicolumn{2}{|c|}{3842} \\
\hline
\end{tabular}

Lecture : dans la génération 1941-1955, les femmes les moins diplômées ont 0,4 fois plus de chances de fumer que les plus diplômées à l'âge de 20 ans.

Champ : population française, femmes âgées de 25 à 69 ans au moment de l'enquête (âgées de 14 à 39 ans pour la cohorte 1971-1985, de 40 à 54 ans pour la cohorte 1956-1970 et de 55 à 65 ans pour la cohorte 1941-1955).

Source : Inpes, Baromètre santé 2010.

Tableau C

Tests de l'égalité des coefficients de l'interaction entre le rang d'éducation et l'âge rétrospectif a (entre 14 et 39 ans)

\begin{tabular}{|l|c|c|c|c|c|c|}
\hline \multirow{2}{*}{ Cohorte } & \multicolumn{2}{|c|}{$1941-1955$} & \multicolumn{2}{c|}{ Cohorte 1956-1970 } & \multicolumn{2}{c|}{ Cohorte 1971-1985 } \\
\cline { 2 - 7 } & Homme & Femme & Homme & Femme & Homme & 25 \\
DF & 25 & 25 & 25 & 25 & 25 & 25 \\
Chi2 & 47,44 & 56,02 & 19,74 & 55,33 & 43,52 & 50,28 \\
P-value & 0,00 & 0,00 & 0,76 & 0,00 & 0,01 & 0,01 \\
\hline
\end{tabular}

Lecture : entre 14 et 39 ans, les inégalités de tabagisme selon le niveau d'éducation présentent une évolution significative avec l'âge pour toutes les cohortes sauf la cohorte intermédiaire chez les hommes.

Champ : population française, hommes âges de 25 à 69 ans au moment de l'enquête (âgés de 14 à 39 ans pour la cohorte 1971-1985,

de 40 à 54 ans pour la cohorte 1956-1970 et de 55 à 65 ans pour la cohorte 1941-1955).

Source : Inpes, Baromètre santé 2010. 